\title{
PARALLELISM AND EQUIDISTANCE IN CLASSICAL DIFFERENTIAL GEOMETRY*
}

BY

\author{
W. C. GRAUSTEIN
}

It is the purpose of this paper to discuss and compare certain concepts bearing on the relationship to one another of two families of curves on a surface in a euclidean space of three dimensions. In the first place, there are set over against one another the concept of parallelism, in the sense of LeviCivita, of one family of curves with respect to another and the concept of equidistance of the first family with respect to the second. Secondly, a measure of the deviation from parallelism of the one family of curves with respect to the other, to which is given the name "angular spread," is compared with a measure of the deviation from equidistance of the first family with respect to the second, which is called "distantial spread."

In addition to the introduction of the concepts in question, Part I contains the fundamental relations between the spreads of each of two families with respect to the other. These relations lead to quantitative results concerning the spreads, as well as to interesting conclusions concerning the connection between parallelism and equidistance.

Part II deals more in detail with the properties of the spreads. In particular, it is found that the angular spread, at a point $P$, of a fixed family of curves with respect to a variable family of curves is represented geometrically by a variable vector at $P$ the locus of whose terminal point is a circle. This fact leads directly to a generalization of Liouville's formula for geodesic curvature which is not only of interest in itself but tends to show that the original formula is essentially a reationship between angular spreads.

Part III bears on the connection between directional derivatives and distantial spreads. It develops an invariant form of Bonnet's formula for geodesic curvature, finds the laws of transformation of the spreads of one system of curves into those of a second, and applies these laws to the study of Tchebycheff systems.

In Part IV, the relationships between the spreads of a system of curves on a surface and the spreads of the corresponding system of curves on the spherical representation of the surface are discussed and application is made of the results to the comparison of parallelism and equidistance on the sur-

* An address presented to the Society at the request of the program committee, November 28 , 1931; received by the editors February 20, 1932. 
face with parallelism and equidistance on the sphere. Fundamental in the treatment are simple geometrical forms of the Codazzi equations of the surface.

It is assumed throughout that the given surface, and the curves on it, are real and analytic.

\section{ANGULAR AND DISTANTIAL SPREADS}

1. Angular spread of a family of directions with respect to a directed curve. We begin by recalling Levi-Civita's concept of parallelism in the case of a surface $S: x_{i}=x_{i}(u, v), i=1,2,3$, in a euclidean space of three dimensions. We shall be interested, not in a parallelism which bears on lengths as well as directions, but in a parallelism of directions only.

At each point $P$ of a directed curve $C$ on $S$ let there be given an oriented direction, tangential, of course, to $S$. Let $1 / \rho$ be the geodesic curvature of $C$, $s$ the directed arc of $C$, and $\omega=\omega(s)$ the directed angle from the directed tangent to $C$ at $P$ to the given oriented direction at $P$. Then the oriented directions in the points of $C$ are parallel to one another in the sense of Levi-Civita if and only if*

$$
\frac{1}{\rho}+\frac{d \omega}{d s}=0
$$

For the sake of later developments, we sketch a proof. Let $\xi$ be the unit vector tangent at $P$ to $C$ and directed in the same sense as $C$, and let $\xi^{\prime}$ be the unit vector in the tangent plane to $S$ at $P$ which is normal to $C$ at $P$ and is so directed that $\xi, \xi^{\prime}$, and the unit vector, $\zeta$, normal to $S$ at $P$ have the same disposition as the axes of coördinates. In terms of $\xi$ and $\xi^{\prime}$, the unit vector $\alpha$ at $P$ which coincides in direction and sense with the given oriented direction at $P$ may be written $\alpha=\xi \cos \omega+\xi^{\prime} \sin \omega$. Hence, when we take account of the well known relations $\dagger$

$$
\frac{d \xi}{d s}=\frac{1}{\rho} \xi^{\prime}+\frac{1}{r} \zeta, \frac{d \xi^{\prime}}{d s}=-\frac{1}{\rho} \xi-\frac{1}{\tau} \zeta, \frac{d \zeta}{d s}=-\frac{1}{r} \xi+\frac{1}{\tau} \xi^{\prime},
$$

where $1 / r$ and $1 / \tau$ are respectively the normal curvature and geodesic torsion of the curve $C$, we find that

* Levi-Civita, Nozione di parallelismo in una varietd qualunque e consequente specificazione geometrica della curvatura Riemanniana, Rendiconti del Circolo Matematico di Palermo, vol. 42 (1917), p. 185.

† See, e.g., Graustein, Invariant methods in classical differential geometry, Bulletin of the American Mathematical Society, vol. 36 (1930), p. 506. 


$$
\frac{d \alpha}{d s}=\left(\frac{1}{\rho}+\frac{d \omega}{d s}\right) \beta+\left(\frac{\cos \omega}{r}-\frac{\sin \omega}{\tau}\right) \zeta,
$$

where $\beta=\widetilde{\zeta \alpha}$. $\dagger$ It follows, then, that the given directions are parallel with respect to the curve $C$ if and only if $1 / \rho+d \omega / d s=0$.

The expression $(\cos \omega) / r-(\sin \omega) / \tau$ vanishes identically if and only if the given directions are conjugate to the directions tangent to $C$, provided it is agreed that at a parabolic point of $S$ two directions are conjugate when and only when at least one of them is the asymptotic direction and that at a planar point each two directions are conjugate. It is clear, then, from (2), that a necessary and sufficient condition that the given directions be absolutely parallel is that they be conjugate to the directions tangent to $C$ and be parallel with respect to $C$ in the sense of Levi-Civita. $\ddagger$

Angular spread. The quantity

$$
\frac{1}{a}=\frac{1}{\rho}+\frac{d \omega}{d s}
$$

formed for the given oriented directions attached to the points of the directed curve $C$, is evidently a measure of the deviation of the directions from parallelism with respect to the curve $C$.

This quantity is called by Struik $\S$ the curvature of the given family of directions with respect to the curve $C$, and, by Bianchi, $\uparrow$ the associate curvature of the family of directions. For reasons which will become apparent later, we prefer to call it the angular spread of the family of directions with respect to $C$.

It is worth while noting that an obvious generalization of the usual definition, in terms of parallelism, of the geodesic curvature of a curve gives rise to angular spread. Let $d$ be the direction of the family at the point $P$ of $C, d^{\prime}$ the direction of the family at a point $P^{\prime}$ of $C$ neighboring to $P$, and $d^{*}$ the direction at $P^{\prime}$ which is parallel with respect to $C$ to the direction $d$ at $P$. Then, if $\Delta \theta$ is the directed angle from $d^{*}$ to $d^{\prime}$ and $\Delta s$ is the directed arc $P P^{\prime}$, the limit, when $\Delta s$ approaches zero, of $\Delta \theta / \Delta s$ is precisely the value, at $P$, of the angular spread, $1 / a: \|$

$\dagger$ Vector notation. If $a: a_{1}, a_{2}, a_{3}$ and $b: b_{1}, b_{2}, b_{3}$ are ordered triples of numbers, their inner and outer products shall be denoted by $(a \mid b)$ and $\widetilde{a b}$ respectively:

$$
(a \mid b): a_{1} b_{1}+a_{2} b_{2}+a_{3} b_{3}, \tilde{a b}: a_{2} b_{3}-a_{3} b_{2}, a_{3} b_{1}-a_{1} b_{3}, a_{1} b_{2}-a_{2} b_{1} \text {. }
$$

† See, e.g., Eisenhart, Riemannian Geometry, p. 167.

\$Struik, Grundzüge der mehrdimensionalen Differentialgeometrie, 1922, p. 77.

I Bianchi, Sul parallelismo vincolato di Levi-Civita nella metrica degli spazi curvi, Rendiconti, Accademia di Napoli, (3), vol. 28 (1922), p. 161.

\| The possibility of this definition of angular spread is pointed out by Bianchi, loc. cit., p. 161. 


$$
\frac{1}{a}=\lim _{\Delta s \rightarrow 0} \frac{\Delta \theta}{\Delta s}
$$

For, if $\alpha+\Delta \alpha$ and $\alpha+\delta \alpha$ are the unit vectors at $P^{\prime}$ having respectively the same directions and senses as $d^{\prime}$ and $d^{*}$, and $\zeta+\Delta \zeta$ is the unit vector normal to the surface at $P^{\prime}$, we have

$$
(\zeta+\Delta \zeta) \sin \Delta \theta=\overline{\alpha+\delta \alpha} \widetilde{\alpha+\Delta \alpha} .
$$

Hence

$$
\zeta \lim _{\Delta s \rightarrow 0} \frac{\Delta \theta}{\Delta s}=\alpha \overline{\frac{d \alpha}{d s}-\frac{\delta \alpha}{\delta s}}=\left(\frac{1}{\rho}+\frac{d \omega}{d s}\right) \tilde{\alpha \beta},
$$

and the conclusion follows.

From (3) or (4) it follows that, when the sense of the curve $C$ is reversed, the angular spread, $1 / a$, changes sign.

2. Angular spread of one family of curves with respect to a second family. Instead of the single directed curve $C$, we shall now assume on $S$ a family of directed curves, which we shall call, individually and collectively, the curves $C$. With reference to the curves $C$ we shall consider a second family of directed curves, the curves $C^{\prime}$. At each point $P:(u, v)$ of $S$ we form the expression

$$
\frac{1}{a}=\frac{1}{\rho}+\frac{\partial \omega}{\partial s}
$$

where $1 / \rho$ is the geodesic curvature at $P$ of the curve $C$ which goes through $P$ and $\partial \omega / \partial s$ is the directional derivative at $P$, in the positive direction of the curve $C$, of the directed angle $\omega$ from this direction to the positive direction, at $P$, of the curve $C^{\prime}$ passing through $P$.

For the points of a curve $C, 1 / a$ is the angular spread, with respect to the curve $C$, of the directions, at the points of $C$, of the curves $C^{\prime}$. It is natural, then, to call $1 / a$ the angular spread of the curves $C^{\prime}$ with respect to the curves $C$, and to say that the curves $C^{\prime}$ are parallel with respect to the curves $C$ (in the sense of Levi-Civita) if and only if $1 / a \equiv 0$.

We agree, further, to say that the curves $C^{\prime}$ are absolutely parallel with respect to the curves $C$ when and only when the directions, at the points of a curve $C$, of the curves $C^{\prime}$ are absolutely parallel to one another for each and every curve $C$. It follows, then, from $\$ 1$, that the curves $C^{\prime}$ are absolutely parallel with respect to the curves $C$ if and only if the family of curves $C^{\prime}$ is conjugate to that of the curves $C$ and the curves $C^{\prime}$ are parallel (in the sense of LeviCivita) with respect to the curves $C$. 
3. Distantial spread of one family of curves with respect to a second family. The angular spread of one family of curves with respect to a second is based on angle. We shall now introduce a "spread" of the one family of curves with respect to the other which is based on distance and which, for want of a better name, we shall call distantial spread.

With respect to a given family of directed curves, which we shall here refer to as the curves $C^{\prime}$, consider a second family of curves $C: f(u, v)=$ const., distinct from the given family. Direct the curves $C$ so that, at each point $P:(u, v)$, the smallest positive directed angle, $\omega$, from the positive direction of the curve $C$ through $P$ to the positive direction of the curve $C^{\prime}$ through $P$ lies between 0 and $\pi$. Mark the distance $d$, measured along an arbitrary curve $C^{\prime}$, between a curve $C: f=f_{0}$ and a neighboring curve $C: f=f_{0}+\Delta f$, and take the logarithmic directional derivative, in the positive direction of the curve $C: f=f_{0}$, of the distance $d$. The limit of this derivative, when $\Delta f$ approaches zero, is defined as the distantial spread, $1 / b^{\prime}$, of the curves $C$ with respect to the curves $C^{\prime}$ :

$$
\frac{1}{b^{\prime}}=\lim _{\Delta f \rightarrow 0} \frac{\partial \log d}{\partial s} .
$$

If $s^{\prime}$ is the directed arc of an individual curve $C^{\prime}$,

$$
d s^{\prime}=\frac{d f}{f_{s^{\prime}}},
$$

where $f_{s^{\prime}}=\partial f / \partial s^{\prime}$ is the directional derivative of $f(u, v)$ in the positive direction of the curve $C^{\prime}$. It follows that

$$
d=|\Delta f|\left[\frac{1}{\left|f_{8^{\prime}}\right|}+\epsilon\right],
$$

where $\epsilon \rightarrow 0$ when $\Delta f \rightarrow 0$. Hence, since $\Delta f$ is a constant, $\dagger$

$$
\frac{1}{b^{\prime}}=-\frac{\partial}{\partial s} \log \left|\frac{\partial f}{\partial s^{\prime}}\right| .
$$

If $1 / b^{\prime} \equiv 0$, then $\partial f / \partial s^{\prime}$ is constant along each curve $C$ and hence is a function of $f(u, v)$. Then $d s^{\prime}$, as given by (7), is the exact differential of a function $F(f)$, and $s^{\prime}=F(f)$ is the common directed arc, measured from a

$\dagger$ The expression in (8) for $1 / b^{\prime}$ (but not the definition from which it follows here) occurs in the theory of directional derivatives on a surface ( $\$ 9)$; the significance of the vanishing of the expression is well known. See Lilienthal, Vorlesungen über Differentialgeometrie, vol. II, p. 228; Graustein, Bulletin paper, loc. cit., p. 498. 
fixed curve $C$, of all the curves $C^{\prime}$. In other words, each two curves $C$ cut segments of equal length from the curves $C^{\prime}$. Accordingly, we say that the curves $C$ are equidistant with respect to the curves $C^{\prime}$.

The foregoing argument may be reversed. Thus, the distantial spread of a family of curves with respect to the given curves $C^{\prime}$ vanishes identically when and only when the curves of the family are equidistant with respect to the curves $C^{\prime}$.

It follows from the agreement concerning the orientation of the curves $C$ that, when the directions of the curves $C^{\prime}$ are reversed, $1 / b^{\prime}$ changes sign.

$A$ second formula for $1 / b^{\prime}$. Since $\left|\partial f / \partial s^{\prime}\right|=\left(\Delta_{1} f\right)^{1 / 2} \sin \omega$, where $\left(\Delta_{1} f\right)^{1 / 2}$ is the magnitude of the gradient of $f(u, v)$,

$$
\frac{1}{b^{\prime}}=-\frac{\partial}{\partial s} \log \left(\Delta_{1} f\right)^{1 / 2}-\frac{\partial}{\partial s} \log \sin \omega .
$$

Lemma. The geodesic curvature, $1 / \rho^{*}$, of the orthogonal trajectories of the directed curves $C: f(u, v)=$ const. is equal to minus the logarithmic rate of change, in the positive directions of the curves $C$, of the length of the gradient of $f(u, v)$ : $1 / \rho^{*}=-\partial\left(\log \left(\Delta_{1} f\right)^{1 / 2}\right) / \partial s$. It is understood that the orthogonal trajectories, $C^{*}$, are so directed that the direction of rotation at each point $P$ from the positive direction of the curve $C$ through $P$ to the positive direction of the curve $C^{*}$ through $P$ is the positive direction of rotation.

By means of the Lemma, we obtain from (9) the following useful formula for $1 / b^{\prime}$ :

$$
\frac{1}{b^{\prime}}=\frac{1}{\rho^{*}}-\frac{\partial}{\partial s} \log \sin \omega .
$$

To establish the Lemma, we note that, if $\alpha$ is the unit vector tangent at an arbitrary point $P$ to the directed curve $C^{*}$ which passes through $P$, the geodesic curvature at $P$ of the curve $C^{*}$, according to Bonnet's formula, is

$$
\frac{1}{\rho^{*}}=\frac{1}{D}\left[\frac{\partial}{\partial u}\left(x_{v} \mid \alpha\right)-\frac{\partial}{\partial v}\left(x_{u} \mid \alpha\right)\right],
$$

where $D^{2}=E G-F^{2}$ is the discriminant of the first fundamental form of $S$. If we assume, as we may, that the function $f(u, v)$ increases when we proceed along a curve $C^{*}$ in the positive direction, then $\left(\Delta_{1} f\right)^{1 / 2} \alpha$ is the gradient at $P$ of $f(u, v)$. Since the projection of this gradient on an oriented direction at $P$ is the directional derivative of $f(u, v)$ in the oriented direction, we conclude that $\left(x_{u} \mid \alpha\right)=f_{u} /\left(\Delta_{1} f\right)^{1 / 2}$ and $\left(x_{v} \mid \alpha\right)=f_{v} /\left(\Delta_{1} f\right)^{1 / 2}$. Thus, the expression (11) takes the form 


$$
\frac{1}{\rho^{*}}=\frac{\partial}{\partial u}\left(\log \frac{1}{\left(\Delta_{1} f\right)^{1 / 2}}\right) \frac{f_{v}}{D\left(\Delta_{1} f\right)^{1 / 2}}-\frac{\partial}{\partial v}\left(\log \frac{1}{\left(\Delta_{1} f\right)^{1 / 2}}\right) \frac{f_{u}}{D\left(\Delta_{1} f\right)^{1 / 2}}
$$

or, when we think of the directed curve $C$ represented parametrically in terms of its arc $s$, the form

$$
\frac{1}{\rho^{*}}=\frac{\partial}{\partial u}\left(\log \frac{1}{\left(\Delta_{1} f\right)^{1 / 2}}\right) \frac{d u}{d s}+\frac{\partial}{\partial v}\left(\log \frac{1}{\left(\Delta_{1} f\right)^{1 / 2}}\right) \frac{d v}{d s}=\frac{\partial}{\partial s} \log \frac{1}{\left(\Delta_{1} f\right)^{1 / 2}}
$$

4. Distantial and angular spreads of the families of a system of curves with respect to one another. Consider a system of curves on $S$ consisting of the family of curves $C$ and the family of curves $C^{\prime}$. Assume that the curves $C$ and the curves $C^{\prime}$ are directed so that $0<\omega<\pi$, where $\omega$ is the angle at $P:(u, v)$ from the directed curve $C$ through $P$ to the directed curve $C^{\prime}$.

Let $1 / a$ and $1 / b$ be respectively the angular spread and the distantial spread of the curves $C^{\prime}$ with respect to the curves $C$, and $1 / a^{\prime}$ and $1 / b^{\prime}$, the angular and distantial spreads of the curves $C$ with respect to the curves $C^{\prime}$. According to (5) and (10),

$$
\begin{aligned}
& \frac{1}{a}=\frac{1}{\rho}+\frac{\partial \omega}{\partial s}, \quad \frac{1}{b}=-\frac{1}{\rho^{\prime *}}+\frac{\partial}{\partial s^{\prime}} \log \sin \omega, \\
& \frac{1}{a^{\prime}}=\frac{1}{\rho^{\prime}}-\frac{\partial \omega}{\partial s^{\prime}}, \quad \frac{1}{b^{\prime}}=\frac{1}{\rho^{*}}-\frac{\partial}{\partial s} \log \sin \omega,
\end{aligned}
$$

where $\partial / \partial s$ and $\partial / \partial s^{\prime}$ denote directional differentiation in the positive directions of the curves $C$ and $C^{\prime}, 1 / \rho$ and $1 / \rho^{\prime}$ the geodesic curvatures of the directed curves $C$ and $C^{\prime}$, and $1 / \rho^{*}$ and $1 / \rho^{\prime *}$ the geodesic curvatures of the orthogonal trajectories $C^{*}$ and $C^{*}$ of the curves $C$ and $C^{\prime}$, when these trajectories have been directed as specified in the Lemma of $\$ 3$.

Liouville's formula for geodesic curvature, applied to the curves $C^{\prime}$ with reference to the curves $C$ and $C^{*}$, and to the curves $C$ with reference to the curves $C^{\prime}$ and $C^{\prime *}$, yields the relations

or

$$
\frac{1}{\rho^{\prime}}-\frac{\partial \omega}{\partial s^{\prime}}=\frac{\cos \omega}{\rho}+\frac{\sin \omega}{\rho^{*}}, \frac{1}{\rho}+\frac{\partial \omega}{\partial s}=\frac{\cos \omega}{\rho^{\prime}}-\frac{\sin \omega}{\rho^{\prime *}},
$$

$$
\frac{\sin \omega}{\rho^{*}}=\frac{1}{a^{\prime}}-\frac{\cos \omega}{\rho}, \quad \frac{\sin \omega}{\rho^{*}}=-\frac{1}{a}+\frac{\cos \omega}{\rho^{\prime}} .
$$

Substituting these values of $1 / \rho^{*}$ and $1 / \rho^{\prime *}$ into (13), we obtain the following expression for $1 / a$ and $1 / a^{\prime}$ in terms of $1 / b$ and $1 / b^{\prime}$, and vice versa: 


$$
\begin{aligned}
& \frac{\sin \omega}{a}=\frac{1}{b}+\frac{\cos \omega}{b^{\prime}}, \\
& \frac{\sin \omega}{a^{\prime}}=\frac{\cos \omega}{b}+\frac{1}{b^{\prime}} \\
& \frac{\sin \omega}{b}=\frac{1}{a}-\frac{\cos \omega}{a^{\prime}}, \\
& \frac{\sin \omega}{b^{\prime}}=-\frac{\cos \omega}{a}+\frac{1}{a^{\prime}} .
\end{aligned}
$$

The implications of these important relations depend on whether or not the curves $C$ and $C^{\prime}$ form an orthogonal system.

Case of an orthogonal system. When $\omega=\pi / 2$, equations (13) and (14) reduce to

$$
\frac{1}{a}=\frac{1}{b}=\frac{1}{\rho}, \quad \frac{1}{a^{\prime}}=\frac{1}{b^{\prime}}=\frac{1}{\rho^{\prime}} .
$$

THEOREM 1. The angular and distantial spreads of a family of curves with respect to the orthogonal trajectories of the family are identical and their common value is the geodesic curvature of the orthogonal trajectories.

The theorem implies that equidistance and parallelism of a family of curves with respect to the orthogonal trajectories of the family are equivalent. This follows also from the well known fact that, for equidistance and parallelism, there is a common necessary and sufficient condition, namely, that the orthogonal trajectories be geodesics.

If, in equations (14), we set $1 / b=1 / a$ and $1 / b^{\prime}=1 / a^{\prime}$ and assume that $1 / a$ and $1 / a^{\prime}$ are not both zero, it follows that $\omega=\pi / 2$.

THEOREM 2. If the angular and distantial spreads of the curves $C^{\prime}$ with respect to the curves $C$ are equal, and the angular and distantial spreads of the curves $C$ with respect to the curves $C^{\prime}$ are equal, and not both these spreads are zero, the curves $C$ and $C^{\prime}$ form an orthogonal system.

Case of a nonorthogonal system. Consider in a plane two distinct analytic families of straight lines. Elementary considerations show that necessary and sufficient conditions that every quadrilateral of the network be a parallelogram are as follows: (a) that each family consist of parallel lines; or (b) that the lines of each family be equidistant with respect to those of the other; or (c) that the lines of one family be parallel, and also equidistant with respect to those of the other; or (d) that the lines of one family be parallel and the lines of the second family be equidistant with respect to those of the first. 
These results admit of complete generalization to the case of the nonorthogonal families of curves $C$ and $C^{\prime}$ on $S$. Here, we have to do with the following four properties of the two families of curves:

Curves $C^{\prime}$ parallel with respect to the curves $C: 1 / a=0$;

$\left\{\right.$ Curves $C^{\prime}$ equidistant with respect to the curves $C: 1 / b=0$;

$\left\{\right.$ Curves $C$ parallel with respect to the curves $C^{\prime}: 1 / a^{\prime}=0$;

Curves $C$ equidistant with respect to the curves $C^{\prime}: 1 / b^{\prime}=0$.

Since $\omega \neq \pi / 2$, it follows from equations (14) that, if any two of the four quantities $1 / a, 1 / b, 1 / a^{\prime}, 1 / b^{\prime}$ are zero, all four are zero. Hence:

THEOREM 3. A nonorthogonal system of curves on a surface which has any two of the properties (A) has the other two also.

In particular, if the curves of each of the families of the system are equidistant with respect to the curves of the other family, then the curves of each family are parallel with respect to the curves of the other family, and conversely. $\dagger$ Thus, the concepts of equidistance and parallelism, which we know to be equivalent when applied to one family of curves of an orthogonal system, are also equivalent for a nonorthogonal system when they are applied to both families of the system.

Again, the theorem says that, if the curves of one family of a nonorthogonal system are both parallel and equidistant with respect to the curves of the other, the curves of the second family are both parallel and equidistant with respect to those of the first.

According to equations (15), the analog of Theorem 3 for an orthogonal system reads as follows.

THEOREM 4. An orthogonal system which has one of the first two of the.properties (A) and one of the last two, has all four properties.

Curves clothing the surface. A system of curves which has the property that the curves of each family are equidistant with respect to the curves of the other family is said to clothe the surface in the sense of Tchebycheff.

From Theorems 3 and 4 we conclude

THEOREM 5. A necessary and sufficient condition that a system of curves clothe a surface is that it have two of the four properties (A), provided merely that, if the system is orthogonal, the two properties belong to different pairs.

$\dagger$ This proposition is well known. The first proof of it was given by Bianchi, Le reti di Tchebychef sulle superficie ed il parallelismo nel senso di Levi-Civita, Bollettino della Unione Matematica Italiana, vol. 1 (1922), pp. 11-16. 
It is to be recalled that, if an orthogonal system clothe a surface, its curves are geodesics and the surface is a developable or a plane.

A surface is a surface of translation $†$ when and only when there exist on it two distinct families of curves such that the curves of each family are absolutely parallel with respect to the curves of the other family. The result of $\S 2$, in conjunction with Theorems 3 and 4 , yields, then, the following proposition.

THEOREM 6. A necessary and sufficient condition that a surface be a surface of translation is that there exist on it a conjugate system which has two of the properties (A), provided that, if the system is orthogonal, the two properties belong to different pairs.

We remark that, if the generators of a translation surface cut at right angles, the surface is a cylinder or a plane.

Quantitative relations between the four spreads. From (14) we obtain the following interesting relations between $1 / a, 1 / a^{\prime}, 1 / b$, and $1 / b^{\prime}$.

THEOREM 7. The angular spreads of two distinct families of curves with respect to one another are equal (or negatives of one another) if and only if the distantial spreads of the two families with respect to one another are equal (or are negatives of one another).

THEOREM 8. The angular and distantial spreads of one family of curves with respect to a second are equal (negatives of one another) if and only if the angular and distantial spreads of the second family with respect to the first are negatives of one another (equal).

The first of these two theorems is valid for every system of curves, whereas the second theorem holds only for a nonorthogonal system and, in the case of an orthogonal system, is to be replaced by Theorem 1 .

We note, finally, that equations (14) are equivalent to the identity

$$
\frac{\sin \alpha^{\prime}}{a}+\frac{\sin \alpha}{a^{\prime}}=\frac{\cos \alpha}{b}+\frac{\cos \alpha^{\prime}}{b^{\prime}},
$$

holding for every two angles $\alpha, \alpha^{\prime}$ whose sum is $\omega$.

\section{Properties of ANgular and distantial spreads}

5. Dual aspects of angular and distantial spreads. Consider two distinct families of curves, the curves $C_{1}$ and the curves $C$, and assume that the curves

$\dagger$ We restrict ourselves here to translation surfaces with real generators, inasmuch as we are dealing only with real curves. Theorem 6 can, however, readily be extended to surfaces of translation with imaginary generators, except perhaps minimal surfaces. 
have been so directed that the directed angle $\alpha$ at $P:(u, v)$ from the directed curve $C_{1}$ through $P$ to the directed curve $C$ through $P$ lies between 0 and $\pi$.

If $1 / a$ and $1 / b$ are respectively the angular and distantial spreads of the curves $C_{1}$ with respect to the curves $C$,

$$
\frac{1}{a}=\frac{1}{\rho}-\frac{\partial \alpha}{\partial s}, \quad \frac{1}{b}=\frac{1}{\rho_{2}}-\frac{\partial}{\partial s_{1}} \log \sin \alpha,
$$

where $\partial / \partial s$ and $\partial / \partial s_{1}$ are the directional derivatives in the positive directions of the curves $C$ and $C_{1}, 1 / \rho$ is the geodesic curvature of the directed curves $C$, and $1 / \rho_{2}$ is the geodesic curvature of the orthogonal trajectories, $C_{2}$, of the curves $C_{1}$, directed as described in the Lemma of $\$ 3$.

We note that the expression for $1 / a$ bears primarily on the curves $C$, whereas that for $1 / b$ pertains essentially to the curves $C_{1}$. For example, it is when the angle $\alpha$ is constant along each of the curves $C$ that $1 / a$ reduces to a geodesic curvature, and this geodesic curvature is that of the curves $C$. On the other hand, it is when $\alpha$ is constant along each curve $C_{1}$ that $1 / b$ reduces to a geodesic curvature and this geodesic curvature is that of the curves $C_{2}$ orthogonal to the curves $C_{1}$.

If we hold the curves $C$ fast and seek all the families of curves $C_{1}$ each of which has a given angular spread with respect to the curves $C$, we obtain a set of families of curves dependent on an arbitrary function of a single variable. Such a set of families of curves is characterized by the property that, if $\alpha(u, v)$ and $\beta(u, v)$ are the directed angles under which two of its families cut the family of curves $C, \beta-\alpha$ is constant along each of the curves $C$. In particular:

THEOREM 9. There is one set of families of curves of the type described each of whose families is a family of parallel curves with respect to the given curves $C$. If the curves $C$ are geodesics, each family of the set cuts the family of curves $C$ under an angle which is constant along the curves $C$.

When we hold the curves $C_{1}$ fast and ask for all the families of curves $C$ with respect to each of which the family of curves $C_{1}$ has a given distantial spread, we obtain a set of families of curves which also depends on an arbitrary function of a single variable. Here, such a set is characterized by the property that, if $\alpha$ and $\beta$ are the directed angles under which two of its families cut the family of curves $C_{1}, \sin \beta / \sin \alpha$ is constant along the curves $C_{1}$. In particular:

THEOREM 10. There is a set of families of the type described with respect to each of whose families the given curves $C_{1}$ are equidistant. If the curves $C_{1}$ are geodesic parallels, each family of the set cuts the family of curves $C_{1}$ under an angle which is constant along the curves $C_{1}$. 
6. Properties of angular spread. A set of families of curves with respect to each of whose families the given family of curves $C_{1}$ has the same distantial spread evidently depends on the curves $C_{1}$. In fact, it is readily shown that there exists no infinite set of families of curves with respect to each of whose families the given family of curves $C_{1}$ has the same distantial spread for each and every choice of the family of curves $C_{1}$.

On the other hand, there do exist sets of families of curves which have the property that each family of a set has the same angular spread with respect to a given family of curves $C$ for each and every choice of the family of curves $C$. These are the one-parameter sets of families of curves characterized by the property that each two families of a set intersect under a constant angle, and which, on account of this property, are known as pencils of families of curves.

THEOREM 11. Every family of a pencil of families of curves has the same angular spread with respect to an arbitrarily chosen, but fixed, family of curves $C$.

By the angular spread of a pencil of families of curves with respect to the curves $C$ we shall mean the angular spread, with respect to the curves $C$, of any family of the pencil.

Angular spread of a pencil. Consider the pencil of families of curves which is determined by the curves $C_{1}$ of $\$ 5$ and hence contains also their orthogonal trajectories, the curves $C_{2}$. By means of Liouville's formula for the geodesic curvature $1 / \rho$ of the curves $C$ in terms of the geodesic curvatures $1 / \rho_{1}$ and $1 / \rho_{2}$ of the curves $C_{1}$ and $C_{2}$, the angular spread, $1 / a$, of this pencil with respect to the curves $C$ is found to have the value

$$
\frac{1}{a}=\frac{\cos \alpha}{\rho_{1}}+\frac{\sin \alpha}{\rho_{2}} .
$$

If the unit vectors at $P:(u, v)$ tangent respectively to the curves $C_{1}, C_{2}$, and $C$ which pass through $P$ are $\xi^{(1)}, \xi^{(2)}$, and $\xi$, it is clear that $\xi=\xi^{(1)} \cos \alpha$ $+\xi^{(2)} \sin \alpha$. Hence, we are led to write $1 / a$ as the scalar product of $\xi$ and the vector

$$
\Gamma=\frac{\xi^{(1)}}{\rho_{1}}+\frac{\xi^{(2)}}{\rho_{2}},
$$

that is, in the form

$$
\frac{1}{a}=(\Gamma \mid \xi)
$$

The vector $\Gamma$ is known as the geodesic curvature vector of the given pen- 
cil of families of curves. It is the same, no matter with respect to which two orthogonal families of curves in the pencil it is constructed. $\dagger$

THEOREM 12. The angular spread, at a point $P$, of a pencil of families of curves with respect to a family of curves $C$ is the projection, on the directed tangent at $P$ to the curve $C$ through $P$, of the geodesic curvature vector of the pencil at $P$.

It follows that the locus of the tip of the vector $(1 / a) \xi$ at $P$, when the family of curves $C$ varies through all possible positions, is a circle described on the geodesic curvature vector at $P$ as a diameter, except when the geodesic curvature vector is a null vector-a case which we exclude for the present. This circle we shall call the indicatrix, at $P$, of the angular spread of the given pencil of families of curves.

Theorem 12 has the following implications.

COROLlary 1. The sum of the squares of the angular spreads of the pencil with respect to two orthogonal families of curves is the same for each two orthogonal families of curves.

COROLlary 2. The maximum absolute value of the angular spread of the pencil, at a point $P$, with respect to a variable family of curves $C$ is the length of the geodesic curvature vector of the pencil at $P$ and is assumed when the curve $C$ through $P$ is tangent to the geodesic curvature vector at $P$.

It is evident from (19) that $1 / a \equiv 0$ for one and only one family of curves $C$, except in the case when $\Gamma$ is a null vector at every point of the surface $S$. But $\Gamma \equiv 0$ if and only if every family of. the pencil consists of geodesics, - a situation which can occur only when $S$ is a developable or a plane.

THEOREM 13. The angular spread of a pencil of families of curves which does not consist entirely of geodesics is zero with respect to a unique family of curves, namely, the family of curves whose direction at each point is perpendicular to the geodesic curvature vector of the pencil at the point. The angular spread of a pencil of families of geodesics is zero with respect to every family of curves.

The first part of the theorem says that there is a unique family of curves $\ddagger$ with respect to which the curves of a given family are parallel, provided the pencil of families determined by the given family does not consist entirely of geodesics.

Suppose, now, that $p$ and $p^{\prime}$ are two distinct pencils of families of curves

† Graustein, Méthodes invariantes dans la géométrie infinitésimale des surfaces, Mémoires de l'Académie Royale de Belgique (Classe des Sciences), (2), vol. 11 (1929), p. 69.

$\ddagger$ The existence of this family, though not its geometrical characterization, is well known; see Bianchi, Sul parallelismo vincolato di Levi-Civita nella metrica degli spazi curvi, Rendiconti, Accademia di Napoli, (3), vol. 28 (1922), p. 168. 
on the surface $S$ and that $\beta(u, v)$ is the directed angle from a family of curves of the pencil $p$ to a family of curves of the pencil $p^{\prime}$. According to (16),

$$
\frac{1}{a^{\prime}}=\frac{1}{a}+\frac{\partial \beta}{\partial s},
$$

where $1 / a$ and $1 / a^{\prime}$ are the angular spreads of $p$ and $p^{\prime}$ with respect to the arbitrary family of curves $C$. Hence, the two pencils have the same angular spread with respect to one and only one family of curves, namely, the family $\beta(u, v)=$ const.

If $S$ is a developable surface or a plane and $p$ is the unique pencil of families of geodesics on it, then $1 / a \equiv 0$ for every family of curves $C$ and (20) reduces to $1 / a^{\prime}=\partial \beta / \partial s$.

THEOREM 14. The angular spread, at a point $P$, of a pencil of families of curves on a developable surface or plane with respect to a family of curves $C$ is the directional derivative at $P$ in the direction of the curve $C$ through $P$, of the directed angle $\beta(u, v)$ from a family of the pencil of families of geodesics to a family of the given pencil.

Comparison of this result with Theorem 12 gives us

THEOREM 15. The geodesic curvature vector of a pencil of families of curves on a developable surface or plane is the gradient of the directed angle under which the pencil cuts the pencil of families of geodesics.

Inasmuch as the curl of the geodesic curvature vector of a pencil of families of curves on a surface $S$ is the negative of the total curvature of $S, \dagger$ it is only on a developable surface or plane that a geodesic curvature vector can be the gradient of a function or that the angular spread of a pencil can be the directional derivative of a function.

7. A generalization of Liouville's formula. We have seen that Liouville's formula for the geodesic curvature, $1 / \rho$, of a family of curves $C$ in terms of the geodesic curvatures, $1 / \rho_{1}$ and $1 / \rho_{2}$, of two orthogonal families of curves $C_{1}$ and $C_{2}$ may be written in the form (17) and that, in this form, it is of prime importance in the study of angular spread. We shall now proceed to show that, in its most general form, the formula is essentially a relationship between angular spreads.

We note, first, an equation which follows directly from the fact that the indicatrix of the angular spread of a pencil of families of curves, or of a single family, with respect to a variable family of curves is a circle. If $1 / a_{1}, 1 / a_{2}$, $1 / a_{3}$ are the angular spreads of a family of directed curves $C^{*}$ with respect to the curves $C_{1}, C_{2}, C_{3}$ of three distinct families of directed curves, then

† Belgian memoir, loc. cit., p. 69. 


$$
\frac{\sin \alpha_{32}}{a_{1}}+\frac{\sin \alpha_{13}}{a_{2}}+\frac{\sin \alpha_{21}}{a_{3}}=0,
$$

where $\alpha_{i j}$ is the directed angle at $P:(u, v)$ from the curve $C_{i}$ through $P$ to the curve $C_{j}$ through $P$.

This fundamental relation enables us to express the angular spread, $1 / a$, of the curves $C^{*}$ with respect to a variable family of curves $C$ in terms of the angular spreads, $1 / a_{1}$ and $1 / a_{2}$, of the curves $C^{*}$ with respect to two fixed families of curves $C_{1}$ and $C_{2}$. Let the directed angle at $P:(u, v)$ from the directed curve $C_{1}$ through $P$ to the directed curve $C_{2}$ through $P$ be $\omega$, let that from the curve $C_{1}$ to the curve $C$ be $\alpha_{1}$, and that from the curve $C$ to the curve $C_{2}$ be $\alpha_{2}$, so that $\alpha_{1}+\alpha_{2}=\omega$. Then

$$
\frac{\sin \omega}{a}=\frac{\sin \alpha_{2}}{a_{1}}+\frac{\sin \alpha_{1}}{a_{2}} .
$$

In particular, when the curves $C_{1}$ and $C_{2}$ form an orthogonal system and are so directed that $\omega=+\pi / 2$, we have

$$
\frac{1}{a}=\frac{\cos \alpha}{a_{1}}+\frac{\sin \alpha}{a_{2}},
$$

where we have replaced $\alpha_{1}$ by $\alpha$.

When the curves $C^{*}$ are taken as coincident with the curves $C$, equations (22) yield expressions for the geodesic curvature, $1 / \rho$, of the curves $C$ in terms of the angular spreads of the curves $C$ with respect to the curves $C_{1}$ and $C_{2}$. In the general case, we have

$$
\frac{\sin \omega}{\rho}=\frac{\sin \alpha_{2}}{a_{1}}+\frac{\sin \alpha_{1}}{a_{2}},
$$

and, when the curves $C_{1}$ and $C_{2}$ form an orthogonal system,

$$
\frac{1}{\rho}=\frac{\cos \alpha}{a_{1}}+\frac{\sin \alpha}{a_{2}} \text {. }
$$

Since $1 / a_{1}=1 / \rho_{1}+\partial \alpha / \partial s_{1}$ and $1 / a_{2}=1 / \rho_{2}+\partial \alpha / \partial s_{2}$, and furthermore, $\left(\partial \alpha / \partial s_{1}\right) \cos \alpha+\left(\partial \alpha / \partial s_{2}\right) \sin \alpha=\partial \alpha / \partial s$, equation (23b) may be rewritten in the form

$$
\frac{1}{\rho}-\frac{\partial \alpha}{\partial s}=\frac{\cos \alpha}{\rho_{1}}+\frac{\sin \alpha}{\rho_{2}},
$$

and this is precisely the formula of Liouville. Thus (23b) is itself Liouville's formula, in the form most suitable for generalization, and the previous formulas are generalizations of it. 
We return to equation (22b) and let the curves $C^{*}$ coincide with the curves $C_{1}$ or the curves $C_{2}$. Then $1 / a$ is the angular spread of the curves $C_{1}$ or the curves $C_{2}$ with respect to the curves $C$ and $1 / a_{1}=1 / \rho_{1}$ and $1 / a_{2}=1 / \rho_{2}$, so that the equation becomes Liouville's formula in its usual form.

8. A relation between a distantial and an angular spread. In $\$ 4$, we saw that the angular and distantial spreads of the curves of a family with respect to their orthogonal trajectories are equal. We proceed to generalize this result, employing for the purpose the notation as to curves and angles introduced in $\$ 4$.

THEOREM 16. If $1 / b^{\prime}$ is the distantial spread of the family of curves $C$ with respect to the family of curves $C^{\prime}$ and $1 / a^{*}$ is the angular spread of the family of curves $C^{\prime *}$ (orthogonal to the curves $C^{\prime}$ ) with respect to the family of curves $C^{*}$ (orthogonal to the curves $C$ ), then

$$
\frac{1}{b^{\prime}}=\frac{1}{a^{*}}-\frac{\partial}{\partial s^{\prime}} \log \tan \frac{\omega}{2} .
$$

Since

$$
\frac{1}{b^{\prime}}=\frac{1}{\rho^{*}}-\frac{\partial}{\partial s} \log \sin \omega, \frac{1}{a^{*}}=\frac{1}{\rho^{*}}+\frac{\partial \omega}{\partial s^{*}}
$$

it follows that

$$
\sin \omega\left(\frac{1}{b^{\prime}}-\frac{1}{a^{*}}\right)=-\cos \omega \frac{\partial \omega}{\partial s}-\sin \omega \frac{\partial \omega}{\partial s^{*}}=-\frac{\partial \omega}{\partial s^{\prime}},
$$

and hence the theorem is established.

If the angle $\omega$ is constant along each curve $C^{\prime}$, then $1 / b^{\prime}=1 / a^{*}$. In particular:

THEOREM 17. If a family of curves $C$ cut a family of curves $C^{\prime}$ under an angle which is constant along each curve $C^{\prime}$, the curves $C$ are equidistant with respect to the curves $C^{\prime}$ if and only if the orthogonal trajectories of the curves $C^{\prime}$ are parallel with respect to the orthogonal trajectories of the curves $C$.

The usual formula for the distantial spread, $1 / b^{\prime}$, of the curves $C$ with respect to the curves $C^{\prime}$ bears primarily on the curves $C$. From (24) we obtain an expression for $1 / b^{\prime}$ which pertains primarily to the curves $C^{\prime}$, namely,

$$
\frac{1}{b^{\prime}}=\frac{\cos \omega}{\rho^{\prime *}}+\frac{\sin \omega}{\rho^{\prime}}-\frac{\partial}{\partial s^{\prime}} \log \tan \frac{\omega}{2} .
$$

When the curves $C^{\prime}$ and $1 / b^{\prime}$ are given, this equation becomes a differential equation for the determination of the angle $\omega$. Hence: 
THEOREM 18. There are infinitely many families of curves which are equidistant with respect to the curves of a given family. The infinity depends on one arbitrary function of a single variable.

This result is to be compared with Theorem 13.

Consider, finally, the totality of families of curves $C$ each of which cuts the given family of curves $C^{\prime}$ under an angle which is constant $(\neq 0)$ along each curve $C^{\prime}$. This totality, $T$, depends on one arbitrary function of a single variable. According to (24), the distantial spread, $1 / b^{\prime}$, of the variable family of curves $C$ of $T$ with respect to the fixed family of curves $C^{\prime}$ is equal to the angular spread, $1 / a^{*}$, of the fixed family of curves $C^{*}$ with respect to the variable family of curves $C^{*}$. But the indicatrix at $P:(u, v)$ of $1 / a^{*}$ is the circle described on that geodesic curvature vector at $P$ as a diameter which is associated with the given curves $C^{\prime}$. This circle, or better, the circle into which it is carried by a rotation about $P$ through the angle $-\pi / 2$, is, then, an indicatrix of the distantial spreads of the families of the totality $T$ with respect to the given family of curves $C^{\prime}$. In particular, if $1 / b^{\prime}, 1 / b_{1}^{\prime}, 1 / b_{2}^{\prime}$ are the distantial spreads, with respect to the curves $C^{\prime}$, of the curves $C$, $C_{1}, C_{2}$ of three families of $T$, then

$$
\frac{\sin \omega}{b^{\prime}}=\frac{\sin \alpha_{2}}{b_{1}^{\prime}}+\frac{\sin \alpha_{1}}{b_{2}^{\prime}},
$$

where $\alpha_{1}, \alpha_{2}, \omega$ have the same meanings as in $\$ 7$, in connection with equation (22a).

\section{RELATIONSHIPS TO MODIFIED DIRECTIONAL DERIVATIVES}

9. Modified directional derivatives $\dagger$. Consider on the surface $S: x=$ $x(u, v)$ two distinct families of curves, the curves $C: \phi(u, v)=$ const. and the curves $C^{\prime}: \psi(u, v)=$ const. Direct the curves so that the functions $\phi$ and $\psi$ increase in the directions which lie respectively to the left of the curves $C$ and $C^{\prime}$, when these curves are traced in their positive senses; assume that the direction of rotation at an arbitrary point $P$ from the directed curve $C$ through $P$ to the directed curve $C^{\prime}$ through $P$ is positive; and denote by $\omega(0<\omega<\pi)$ the angle from the first of these curves to the second.

For the purpose in view it is convenient to introduce the quantities

$$
A=\frac{\phi_{u}}{\phi_{s^{\prime}}}, \quad B=\frac{\phi_{v}}{\phi_{s^{\prime}}}, \quad A^{\prime}=\frac{\psi_{u}}{\psi_{s}}, \quad B^{\prime}=\frac{\psi_{v}}{\psi_{s}},
$$

where, for example, $\phi_{u}=\partial \phi / \partial u$ and $\phi_{s^{\prime}}=\partial \phi / \partial s^{\prime}$.

$\dagger$ Graustein, Bulletin paper, loc. cit., p. 500, ff.; also, Belgian memoir. The treatment here, though condensed, is simpler than in the papers cited. 
Equivalent definitions of $A, B, A^{\prime}, B^{\prime}$ are furnished by the relations

$$
d \phi=\frac{\partial \phi}{\partial s^{\prime}}(A d u+B d v), \quad d \psi=\frac{\partial \psi}{\partial s}\left(A^{\prime} d u+B^{\prime} d v\right) .
$$

Hence, the differential equations $A d u+B d v=0$ and $A^{\prime} d u+B^{\prime} d v=0$ represent respectively the families of curves $C$ and $C^{\prime}$.

Inasmuch as the differentials of arc, $d s$ and $d s^{\prime}$, of individual curves $C$ and $C^{\prime}$ are given by $d s=d \psi / \psi_{s}$ and $d s^{\prime}=d \phi / \phi_{s^{\prime}}$, we have

$$
d s=A^{\prime} d u+B^{\prime} d v, d s^{\prime}=A d u+B d v .
$$

It is readily shown that, if $\chi(u, v)$ is an arbitrary function,

$$
d \chi=\frac{\partial \chi}{\partial s} d s+\frac{\partial \chi}{\partial s^{\prime}} d s^{\prime} .
$$

Hence it follows that

$$
\begin{array}{ll}
\chi_{u}=A^{\prime} \frac{\partial \chi}{\partial s}+A \frac{\partial \chi}{\partial s^{\prime}}, & \chi_{v}=B^{\prime} \frac{\partial \chi}{\partial s}+B \frac{\partial \chi}{\partial s^{\prime}} \\
\frac{\partial \chi}{\partial s}=\frac{1}{H}\left(B \chi_{u}-A \chi_{v}\right), & \frac{\partial \chi}{\partial s^{\prime}}=-\frac{1}{H}\left(B^{\prime} \chi_{u}-A^{\prime} \chi_{v}\right),
\end{array}
$$

where

$$
H=A^{\prime} B-A B^{\prime} .
$$

In particular, if $\xi=\partial x / \partial s$ and $\xi^{\prime}=\partial x / \partial s^{\prime}$ are the unit vectors at $P$ tangent respectively to the curves $C$ and $C^{\prime}$ through $P$ and directed in the same senses as these curves, we have

$$
\begin{aligned}
x_{u} & =A^{\prime} \xi+A \xi^{\prime}, & x_{v} & =B^{\prime} \xi+B \xi^{\prime}, \\
\xi & =\frac{1}{H}\left(B x_{u}-A x_{v}\right), & \xi^{\prime} & =-\frac{1}{H}\left(B^{\prime} x_{u}-A^{\prime} x_{v}\right) .
\end{aligned}
$$

If the latter expressions are substituted in $\widetilde{\xi \xi^{\prime}}=\zeta \sin \omega$, where $\zeta$ is the unit vector normal to $S$ at $P$, we find for $B$ the value

$$
H=D \csc \omega,
$$

where $D^{2}=E G-F^{2}$ is the discriminant of the first fundamental form of $S$.

We are now in a position to find the values, in terms of $A, B, A^{\prime}, B^{\prime}$, of the distantial spreads, $1 / b$ and $1 / b^{\prime}$, of the curves $C^{\prime}$ and $C$ with respect to the curves $C$ and $C^{\prime}$. We have, namely, 


$$
\frac{1}{b}=\frac{1}{H}\left(B_{u}^{\prime}-A_{v}^{\prime}\right), \frac{1}{b^{\prime}}=\frac{1}{H}\left(B_{u}-A_{v}\right) .
$$

For $1 / b^{\prime}$, for example, is given, according to (8), by

Hence

$$
\frac{1}{b^{\prime}}=-\frac{\partial}{\partial s} \log \frac{\partial \phi}{\partial s^{\prime}} \text {. }
$$

$$
\frac{1}{b^{\prime}}=-\frac{1}{H}\left(B \frac{\partial}{\partial u} \log \phi_{8^{\prime}}-A \frac{\partial}{\partial v} \log \phi_{8^{\prime}}\right) .
$$

Replacing $\phi_{\boldsymbol{s}^{\prime}}$ by $\phi_{v} / B$ in the first term in the parenthesis, and by $\phi_{u} / A$ in the second term, and simplifying, we obtain the desired expression.

By means of equations (29), we find that the fundamental relation $\left(\chi_{u}\right)_{v}=\left(\chi_{v}\right)_{u}$, when expressed in terms of the directional derivatives along the curves $C$ and $C^{\prime}$, takes the following invariant form:

$$
-\frac{1}{H}\left[\left(B^{\prime} \chi_{s}\right)_{u}-\left(A^{\prime} \chi_{s}\right)_{v}\right]=\frac{1}{H}\left[\left(B \chi_{s^{\prime}}\right)_{u}-\left(A \chi_{s^{\prime}}\right)_{v}\right]
$$

We are thus led to introduce modified directional derivatives, defined for an arbitrary function, $F(u, v)$, as follows:

$$
\frac{\nabla F}{\nabla s}=\frac{1}{H}\left[(B F)_{u}-(A F)_{v}\right], \frac{\nabla F}{\nabla s^{\prime}}=-\frac{1}{H}\left[\left(B^{\prime} F\right)_{u}-\left(A^{\prime} F\right)_{v}\right] .
$$

The foregoing relation then takes the simple form

$$
\frac{\nabla}{\nabla s^{\prime}} \frac{\partial \chi}{\partial s}=\frac{\nabla}{\nabla s} \frac{\partial \chi}{\partial s^{\prime}}
$$

The modified derivative of the sum of two functions obeys the usual law. However, in the case of a product, it is clear from (29) and (32a) that we have

$$
\frac{\nabla\left(\chi_{1} \chi_{2}\right)}{\nabla s}=\chi_{1} \frac{\nabla \chi_{2}}{\nabla s}+\chi_{2} \frac{\partial \chi_{1}}{\partial s}=\chi_{1} \frac{\partial \chi_{2}}{\partial s}+\chi_{2} \frac{\nabla \chi_{1}}{\nabla s} .
$$

In particular, it follows that

$$
\frac{\nabla \chi}{\nabla s}=\frac{\partial \chi}{\partial s}+\chi \frac{\nabla(1)}{\nabla s}, \quad \frac{\nabla \chi}{\nabla s^{\prime}}=\frac{\partial \chi}{\partial s^{\prime}}+\chi \frac{\nabla(1)}{\nabla s^{\prime}} .
$$

But, according to (31),

$$
\frac{1}{b}=-\frac{\nabla(1)}{\nabla s^{\prime}}, \quad \frac{1}{b^{\prime}}=\frac{\nabla(1)}{\nabla s} .
$$


Hence

$$
\frac{\nabla \chi}{\nabla s}=\frac{\partial \chi}{\partial s}+\frac{\chi}{b^{\prime}}, \quad \frac{\nabla \chi}{\nabla s^{\prime}}=\frac{\partial \chi}{\partial s^{\prime}}-\frac{\chi}{b} .
$$

10. An invariant form of Bonnet's formula for geodesic curvature. Bonnet's formula for the geodesic curvature, $1 / \rho^{*}$, of a family of directed curves $C^{*}$ is

$$
\frac{1}{\rho^{*}}=\frac{1}{D}\left[\frac{\partial}{\partial u}\left(x_{v} \mid \xi^{*}\right)-\frac{\partial}{\partial v}\left(x_{u} \mid \xi^{*}\right)\right],
$$

where $\xi^{*}$ is the unit vector at $P:(u, v)$ tangent to the curve $C^{*}$ which passes through $P$. When we set for $x_{u}$ and $x_{v}$ their values in terms of $\xi$ and $\xi^{\prime}$, as given by (30), and take account of the definitions (32a) of the modified directional derivatives along the curves $C$ and $C^{\prime}$, the formula takes on the invariant form

or

$$
\frac{\sin \omega}{\rho^{*}}=\frac{\nabla}{\nabla s}\left(\xi^{\prime} \mid \xi^{*}\right)-\frac{\nabla}{\nabla s^{\prime}}\left(\xi \mid \xi^{*}\right),
$$

$$
\frac{\sin \omega}{\rho^{*}}=\frac{\nabla \cos \alpha^{\prime}}{\nabla s}-\frac{\nabla \cos \alpha}{\nabla s^{\prime}},
$$

where $\alpha$ is the angle at $P$ from the curve $C$ to the curve $C^{*}$ and $\alpha^{\prime}$ that from the curve $C^{*}$ to the curve $C^{\prime}$, and $\alpha+\alpha^{\prime}=\omega$.

Expanding (34b), we get

$$
\frac{\sin \omega}{\rho^{*}}=\frac{\cos \alpha}{b}+\frac{\cos \alpha^{\prime}}{b^{\prime}}-\sin \alpha^{\prime} \frac{\partial \alpha^{\prime}}{\partial s}+\sin \alpha \frac{\partial \alpha}{\partial s^{\prime}},
$$

or, by virtue of the relation at the end of $\$ 4$,

$$
\frac{\sin \omega}{\rho^{*}}=\frac{\sin \alpha^{\prime}}{a}+\frac{\sin \alpha}{a^{\prime}}-\sin \alpha^{\prime} \frac{\partial \alpha^{\prime}}{\partial s}+\sin \alpha \frac{\partial \alpha}{\partial s^{\prime}} \text {. }
$$

We may also write the value of $1 / \rho^{*}$ in terms of the geodesic curvatures of the curves $C$ and $C^{\prime}$, but the resulting formula would be identical, essentially, with (23a).

The formulas for the angular spread, $1 / a^{*}$, of the curves $C$ with respect to the curves $C^{*}$ which correspond to (35) are

$$
\begin{aligned}
& \frac{\sin \omega}{a^{*}}=\frac{\cos \alpha}{b}+\frac{\cos \alpha^{\prime}}{b^{\prime}}-\sin \alpha^{\prime} \frac{\partial \omega}{\partial s}, \\
& \frac{\sin \omega}{a^{*}}=\frac{\sin \alpha^{\prime}}{a}+\frac{\sin \alpha}{a^{\prime}}-\sin \alpha^{\prime} \frac{\partial \omega}{\partial s} .
\end{aligned}
$$


The first of these is obtained from (35a) by use of the relation

$$
\sin \omega \frac{\partial \alpha}{\partial s^{*}}=\sin \alpha^{\prime} \frac{\partial \alpha}{\partial s}+\sin \alpha \frac{\partial \alpha}{\partial s^{\prime}},
$$

between the directional derivatives along the curves $C, C^{\prime}$, and $C^{*}$.

Corresponding formulas for distantial spreads. If $0<\alpha<\pi$, that is, if the direction of rotation at $P$ from $\xi$ to $\xi^{*}$ is the positive direction, the distantial spread, $1 / b^{*}$, of the curves $C^{*}$ with respect to the curves $C$ has, according to (10), the value

$$
\frac{1}{b^{*}}=-\frac{1}{\bar{\rho}^{*}}+\frac{\partial}{\partial s^{*}} \log \sin \alpha,
$$

where $1 / \bar{\rho}^{*}$ is the geodesic curvature of the orthogonal trajectories of the curves $C^{*}$, directed as prescribed in the lemma of $\S 3$. Replacing $1 / \bar{\rho}^{*}$ by its value, as given by $(34 \mathrm{~b})$, we get

$$
\frac{\sin \omega}{b^{*}}=-\frac{\nabla \sin \alpha^{\prime}}{\nabla s}-\frac{\nabla \sin \alpha}{\nabla s}+\sin \omega \frac{\partial}{\partial s^{*}} \log \sin \alpha,
$$

or

$$
\frac{\sin \omega}{b^{*}}=\frac{\sin \alpha}{b}-\frac{\sin \alpha^{\prime}}{b^{\prime}}+\sin \alpha^{\prime} \frac{\partial}{\partial s} \log \frac{\sin \alpha}{\sin \alpha^{\prime}} .
$$

An application of distantial spreads. If we assume not only that $0<\alpha<\pi$, but also that $0<\alpha^{\prime}<\pi$, and consider, in conjunction with (36), the formula

$$
\frac{1}{b^{* \prime}}=\frac{1}{\bar{\rho}^{*}}-\frac{\partial}{\partial s^{*}} \log \sin \alpha^{\prime}
$$

for the distantial spread of the curves $C^{*}$ with respect to the curves $C^{\prime}$, we obtain immediately the relation

$$
\frac{1}{b^{*}}+\frac{1}{b^{* \prime}}=\frac{\partial}{\partial s^{*}} \log \frac{\sin \alpha}{\sin \alpha^{\prime}} .
$$

Geometrically, the assumptions to which this formula is subject mean that the tangent to the curve $C^{*}$ at $P:(u, v)$ divides the directed tangents to the curves $C$ and $C^{\prime}$ internally. If this division is external, for example, if $0<\alpha<\pi$, but $-\pi<\alpha^{\prime}<0$, then (38a) is replaced by

$$
\frac{1}{b^{*}}-\frac{1}{b^{* \prime}}=\frac{\partial}{\partial s^{*}} \log \left(-\frac{\sin \alpha}{\sin \alpha^{\prime}}\right) \text {. }
$$


It is evident that in either case the numerical value of $\sin \alpha / \sin \alpha^{\prime}$ is the ratio in which the tangent $t^{*}$ to $C^{*}$ at $P$ divides the tangents $t$ and $t^{\prime}$ to $C$ and $C^{\prime}$ at $P$, that is, the quotient of the distances from an arbitrary point on $t^{*}$ to $t$ and $t^{\prime}$. It is natural then to speak of this ratio as the ratio in which the curves $C^{*}$ divide the curves $C$ and $C^{\prime}$.

THEOREM 19. The family of curves $C^{*}$ divides the families of directed curves $C$ and $C^{\prime}$ externally (internally) in a ratio which is constant along each curve $C^{*}$ if and only if the distantial spreads of the curves $C^{*}$ with respect to the curves $C$ and $C^{\prime}$ are equal (negatives of one another). $\dagger$

11. Formulas of transformation of spreads. We propose to find the laws of transformation from the angular (distantial) spreads of the families of one system of curves with respect to each other to the angular (distantial) spreads of the families of a second system with respect to one another.

Let the two systems of curves consist respectively of the families of directed curves $C, C^{\prime}$ and $C^{*}, C^{* \prime}$. Assume that, in the case of both systems, the angle of rotation at $P:(u, v)$ from the directed curve of the first family through $P$ to the directed curve of the second family through $P$ is positive, and denote the angle, between 0 and $\pi$, from the first of these curves to the second by $\omega$, in the case of the first system, and by $\omega^{*}$, in the case of the second. Further, let $\alpha$ be the angle at $P$ from the directed curve $C$ to the directed curve $C^{*}$, and $\alpha^{\prime}$ that from the directed curve $C^{*}$ to the directed curve $C^{\prime}$. Similarly, let $\beta$ be the angle from $C$ to $C^{* \prime}$, and $\beta^{\prime}$ that from $C^{* \prime}$ to $C^{\prime}$. Evidently, $\alpha, \alpha^{\prime}, \beta, \beta^{\prime}$ may be so chosen that $\alpha+\alpha^{\prime}=\beta+\beta^{\prime}=\omega$ and $\beta-\alpha=\alpha^{\prime}-\beta^{\prime}=\omega^{*}$.

The formulas of transformation from the angular spreads, $1 / a$ and $1 / a^{\prime}$, of the curves $C^{\prime}$ and $C$ with respect to the curves $C$ and $C^{\prime}$ to the angular spreads, $1 / a^{*}$ and $1 / a^{* \prime}$, of the curves $C^{* \prime}$ and $C^{*}$ with respect to the curves $C^{*}$ and $C^{* \prime}$ are

$$
\begin{aligned}
& \frac{\sin \omega}{a^{*}}=\frac{\sin \alpha^{\prime}}{a}+\frac{\sin \alpha}{a^{\prime}}-\sin \alpha^{\prime} \frac{\partial \beta^{\prime}}{\partial s}+\sin \alpha \frac{\partial \beta}{\partial s^{\prime}}, \\
& \frac{\sin \omega}{a^{* \prime}}=\frac{\sin \beta^{\prime}}{a}+\frac{\sin \beta}{a^{\prime}}-\sin \beta^{\prime} \frac{\partial \alpha^{\prime}}{\partial s}+\sin \beta \frac{\partial \alpha}{\partial s^{\prime}} .
\end{aligned}
$$

When $\omega=\pi / 2$, then $1 / a=1 / \rho$ and $1 / a^{\prime}=1 / \rho^{\prime}$ and equations (39) become the formulas of transformation from the geodesic curvatures of the curves of an

† The "dual" theorem says that the angle under which the curves $C$ and $C^{\prime}$ intersect is constant along the curves $C *$ when and only when the curves $C$ and $C^{\prime}$ have the same angular spreads with respect to the curves $C *$. See $\$ 5$. 
orthogonal system to the angular spreads of the families of an arbitrary system:

$$
\frac{1}{a^{*}}=\frac{\cos \alpha}{\rho}+\frac{\sin \alpha}{\rho^{\prime}}+\frac{\partial \beta}{\partial s^{*}}, \quad \frac{1}{a^{* \prime}}=\frac{\cos \beta}{\rho}+\frac{\sin \beta}{\rho^{\prime}}+\frac{\partial \alpha}{\partial s^{* \prime}} .
$$

To establish, say, the first of equations (39), we have merely to add corresponding sides of equation (35b) and the equation

$$
\sin \omega \frac{\partial \omega^{*}}{\partial s^{*}}=\sin \alpha^{\prime} \frac{\partial \omega^{*}}{\partial s}+\sin \alpha \frac{\partial \omega^{*}}{\partial s^{\prime}}
$$

and take account of the relations $\beta-\alpha=\omega^{*}$ and $\alpha^{\prime}-\beta^{\prime}=\omega^{*}$.

The distantial spreads, $1 / b^{*}$ and $1 / b^{* \prime}$, of the curves $C^{* \prime}$ and $C^{*}$ with respect to the curves $C^{*}$ and $C^{* \prime}$ are given by the formulas

$$
\frac{1}{b^{*}}=-\frac{1}{\bar{\rho}^{*}}+\frac{\partial}{\partial s^{* \prime}} \log \sin \omega^{*}, \quad \frac{1}{b^{* \prime}}=\frac{1}{\bar{\rho}^{* \prime}}-\frac{\partial}{\partial s^{*}} \log \sin \omega^{*},
$$

where $1 / \bar{\rho}^{*}$ and $1 / \bar{\rho}^{* \prime}$ are the geodesic curvatures of the directed orthogonal trajectories of the curves $C^{*}$ and $C^{* \prime}$, respectively. Using the values given for these geodesic curvatures by the invariant form of the formula of Bonnet, we get

$$
\begin{aligned}
& \frac{\sin \omega}{b^{*}}=\frac{\sin \beta}{b}-\frac{\sin \beta^{\prime}}{b^{\prime}}-\frac{\partial \sin \beta^{\prime}}{\partial s}-\frac{\partial \sin \beta}{\partial s^{\prime}}+\sin \omega \frac{\partial}{\partial s^{* \prime}} \log \sin \omega^{*} \\
& \frac{\sin \omega}{b^{* \prime}}=-\frac{\sin \alpha}{b}+\frac{\sin \alpha^{\prime}}{b^{\prime}}+\frac{\partial \sin \alpha^{\prime}}{\partial s}+\frac{\partial \sin \alpha}{\partial s^{\prime}}-\sin \omega \frac{\partial}{\partial s^{*}} \log \sin \omega^{*},
\end{aligned}
$$

as the formulas of transformation of $1 / b, 1 / b^{\prime}$ into $1 / b^{*}, 1 / b^{* \prime}$.

12. Systems of Tchebycheff. The formulas of the previous paragraph enable us to write differential equations for the determination of the systems of Tchebycheff on a surface which are simple in form and yield new information.

Let the surface be thought of as referred to a fixed orthogonal system of curves, $C$ and $C^{\prime}$. The arbitrary system of curves $C^{*}$ and $C^{* \prime}$ clothe the surface, according to Theorem 5 , if and only if $1 / a^{*}=0$ and $1 / a^{* \prime}=0$. But equations (40) say that these conditions are satisfied when and only when the angles $\alpha$ and $\beta$, which fix the positions of the curves $C^{*}$ and $C^{* \prime}$ with respect to the curves $C$ and $C^{\prime}$, satisfy the differential equations

$$
\frac{\partial \beta}{\partial s^{*}}+\frac{\cos \alpha}{\rho}+\frac{\sin \alpha}{\rho^{\prime}}=0, \quad \frac{\partial \alpha}{\partial s^{* \prime}}+\frac{\cos \beta}{\rho}+\frac{\sin \beta}{\rho^{\prime}}=0,
$$


where

$$
\frac{\partial \beta}{\partial s^{*}}=\cos \alpha \frac{\partial \beta}{\partial s}+\sin \alpha \frac{\partial \beta}{\partial s^{\prime}}, \quad \frac{\partial \alpha}{\partial s^{* \prime}}=\cos \beta \frac{\partial \alpha}{\partial s}+\sin \beta \frac{\partial \alpha}{\partial s^{\prime}} .
$$

Thus, we have, for the determination of the systems of Tchebycheff on a surface, two partial differential equations of the first order in two dependent and two independent variables. $\dagger$

Curves clothing a developable surface. It is clear geometrically that any two families. of geodesics chosen from the pencil of families of geodesics on a developable surface or plane clothe the surface. In seeking a characterization of the other systems of curves clothing a developable or a plane, we assume that the curves $C$ and $C^{\prime}$ constitute two orthogonal families of geodesics. Equations (42) then become

$$
\frac{\partial \beta}{\partial s^{*}}=0, \frac{\partial \alpha}{\partial s^{* \prime}}=0,
$$

and yield the following result.

TheOREM 20. A necessary and sufficient condition that two families of curves on a developable surface or plane clothe the surface is that the angle which fixes the position of each family with respect to a family of geodesics chosen from the pencil of families of geodesics be constant along the curves of the other family.

Let us now recall that the Gauss equation of a surface, referred to an arbitrary system of curves $C$ and $C^{\prime}$, may be written in the form $\neq$

$$
K \sin \omega=\frac{\nabla}{\nabla s^{\prime}}\left(\frac{1}{\rho}\right)-\frac{\nabla}{\nabla s}\left(\frac{1}{a^{\prime}}\right)=\frac{\nabla}{\nabla s^{\prime}}\left(\frac{1}{a}\right)-\frac{\nabla}{\nabla s}\left(\frac{1}{\rho^{\prime}}\right) .
$$

† These equations may be compared with those of Servant; see Bianchi, Lezioni di Geometria Differenziale, 3d edition, vol. 1, p. 157.

The equations corresponding to (42) in the case in which the system of curves $C$ and $C^{\prime}$ to which $S$ is referred is arbitrary may be obtained by setting $1 / a^{*}$ and $1 / a^{*^{\prime}}$ equal to zero in equations (39). There is, however, another approach to this problem which yields the desired equations in a more elegant form. It follows from (31) that the curves $C *$ and $C *^{\prime}$ clothe the surface if and only if their elements of arc, $d s^{*}$ and $d s^{*^{\prime}}$, are exact differentials. But the expressions for $d s^{*}$ and $d s^{*}$, in terms of the elements of arc, $d s$ and $d s^{\prime}$, of the curves $C$ and $C^{\prime}$ are

$$
d s^{*} \sin \omega^{*}=d s \sin \beta-d s^{\prime} \sin \beta^{\prime}, \quad d s^{*} \sin \omega^{*}=-d s \sin \alpha+d s^{\prime} \sin \alpha^{\prime},
$$

where $\alpha, \alpha^{\prime}, \beta, \beta^{\prime}$ are the angles which fix the curves $C^{*}, C^{*}$ with reference of the curves $C, C^{\prime}$, and these expressions are exact differentials, by (33), if and only if

$$
\frac{\nabla}{\nabla s} \frac{\sin \alpha^{\prime}}{\sin \omega^{*}}+\frac{\nabla}{\nabla s^{\prime}} \frac{\sin \alpha}{\sin \omega^{*}}=0, \frac{\nabla}{\nabla s} \frac{\sin \beta^{\prime}}{\sin \omega^{*}}+\frac{\nabla}{\nabla s^{\prime}} \frac{\sin \beta}{\sin \omega^{*}}=0 .
$$

These, then, are the required differential equations.

$\ddagger$ Belgian memoir, loc. cit., p. 80 . 
Assume that the curves $C$ and $C^{\prime}$ clothe the surface. Then, since $1 / b=0$ and $1 / b^{\prime}=0$, it follows from (32b) that the modified directional derivatives become ordinary directional derivatives. Moreover, by (31), $d s=A^{\prime} d u+B^{\prime} d v$ and $d s^{\prime}=A d u+B d v$ are exact differentials of functions $s=s(u, v)$ and $s^{\prime}=$ $s^{\prime}(u, v)$ which are the common arcs of the curves $C$ and $C^{\prime}$, respectively. Hence, $s$ and $s^{\prime}$ are parameters on the surface and the directional derivatives in equation (44) become ordinary partial derivatives with respect to these parameters.

Since $1 / a=0$ and $1 / a^{\prime}=0,(44)$ now becomes

$$
K \sin \omega=\frac{\partial}{\partial s^{\prime}}\left(\frac{1}{\rho}\right)=-\frac{\partial}{\partial s}\left(\frac{1}{\rho^{\prime}}\right),
$$

and yields the following result.

THEOREM 21. If the geodesic curvature of one family of a system of curves which clothes a surface is constant along the curves of the second family, the surface is a developable or a plane, and conversely.

Since $1 / \rho+\partial \omega / \partial s=0$ and $1 / \rho^{\prime}-\partial \omega / \partial s^{\prime}=0$, equation (45) may also be written in the form

$$
K \sin \omega=-\frac{\partial^{2} \omega}{\partial s^{\prime} \partial s} .
$$

Hence, the angle of intersection of two families of curves which clothe a surface may be written as the sum of two functions one of which is constant along the curves of the one family and the other constant along the curves of the other family when and only when the surface is a developable or a plane. $\dagger$

\section{RELATIONSHIPS BETWEEN SPREADS ON A SURFACE AND THE CORRESPONDING SPREADS ON THE GAUSS SPHERE}

13. Geodesic curvature of a family of curves on the Gauss sphere. We assume now that our surface $S: x=x(u, v)$ is not a developable surface or a plane, and think of it as referred to an arbitrary system of curves, $C$ and $C^{\prime}$.

According to Bonnet's formula, the geodesic curvature, $1 / \mathfrak{r}$, of a family of directed curves on the spherical representation, $\zeta=\zeta(u, v)$, of $S$ has the value

$$
\frac{1}{\mathfrak{r}}=\frac{1}{\mathcal{D}}\left[\frac{\partial}{\partial u}\left(\zeta_{v} \mid \gamma\right)-\frac{\partial}{\partial v}\left(\zeta_{u} \mid \gamma\right)\right]
$$

where $\gamma$ is the unit vector, at the point $P:(u, v)$ on the sphere, tangent to the 
curve of the family which goes through $\mathcal{P}$, and $\mathcal{D}$ is the positive square root of the discriminant of the linear element of the spherical representation.

Substituting for $\zeta_{u}$ and $\zeta_{v}$ their values in terms of $\partial \zeta / \partial s$ and $\partial \zeta / \partial s^{\prime}$, namely,

$$
\zeta_{u}=A^{\prime} \frac{\partial \zeta}{\partial s}+A \frac{\partial \zeta}{\partial s^{\prime}}, \quad \zeta_{v}=B^{\prime} \frac{\partial \zeta}{\partial s}+B \frac{\partial \zeta}{\partial s^{\prime}},
$$

and taking account of the definitions (32a) of the modified directional derivatives along the curves $C$ and $C^{\prime}$, we find that

$$
\frac{1}{\mathfrak{r}}=\frac{H}{\mathcal{D}}\left[\frac{\nabla}{\nabla s}\left(\frac{\partial \zeta}{\partial s^{\prime}} \mid \gamma\right)-\frac{\nabla}{\nabla s^{\prime}}\left(\frac{\partial \zeta}{\partial s} \mid \gamma\right)\right] .
$$

Recalling that $H=D \csc \omega$ and that $\mathcal{D}=\eta K D$, where $\eta=1$ or $\eta=-1$, according as $K>0$ or $K<0$, we obtain, as the final form of $1 / \mathfrak{r}$,

$$
\frac{\sin \omega}{\mathfrak{r}}=\frac{\eta}{K}\left[\frac{\nabla}{\nabla s}\left(\frac{\partial \zeta}{\partial s^{\prime}} \mid \gamma\right)-\frac{\nabla}{\nabla s^{\prime}}\left(\frac{\partial \zeta}{\partial s} \mid \gamma\right)\right] .
$$

14. Spherical representation of a conjugate system. When the curves $C$ and $C^{\prime}$ on $S$ form a conjugate system, $K=\csc ^{2} \omega /\left(r r^{\prime}\right) \dagger$, where $1 / r$ and $1 / r^{\prime}$ are the normal curvatures of the curves $C$ and $C^{\prime}$, respectively. Hence, formula (46) becomes

$$
\frac{1}{\mathfrak{r}}=\epsilon \epsilon^{\prime} r r^{\prime} \sin \omega\left[\frac{\nabla}{\nabla s}\left(\frac{\partial \zeta}{\partial s^{\prime}} \mid \gamma\right)-\frac{\nabla}{\nabla s^{\prime}}\left(\frac{\partial \zeta}{\partial s} \mid \gamma\right)\right],
$$

where $\epsilon=1$ or $\epsilon=-1$ according as $r>0$ or $r<0$, and $\epsilon^{\prime}= \pm 1$ according as $r^{\prime} \gtrless 0$.

To facilitate the work which follows, we introduce, in addition to the unit vectors $\xi$ and $\xi^{\prime}$ which are tangent at $P:(u, v)$ to the directed curves $C$ and $C^{\prime}$ which pass through $P$, the vectors $\bar{\xi}$ and $\bar{\xi}^{\prime}$ lying in the tangent plane at $P$ and advanced by $+\pi / 2$ over the vectors $\xi$ and $\xi^{\prime}$ respectively.

In terms of these vectors, we havef

$$
\frac{\partial \zeta}{\partial s}=\frac{\csc \omega}{r} \overline{\xi^{\prime}}, \quad \frac{\partial \zeta}{\partial s^{\prime}}=-{\frac{\csc \omega}{r^{\prime}}}^{\circ} \bar{\xi} .
$$

Consequently, if we take as the positive directions on the curves $\mathcal{C}$ and $\mathcal{C}^{\prime}$ on the sphere which represent the curves $C$ and $C^{\prime}$ on $S$ the directions which correspond to the positive directions on the curves $C$ and $C^{\prime}$, the unit vectors, 
$\gamma$ and $\gamma^{\prime}$, at $\Phi:(u, v)$ which are tangent to the curves $\mathcal{C}$ and $\mathcal{C}^{\prime}$ passing through $\mathcal{P}$ are

$$
\gamma=\epsilon \overline{\xi^{\prime}}, \quad \gamma^{\prime}=-\epsilon^{\prime} \bar{\xi} .
$$

By means of (47), with the help of (48) and (49), we may now compute the geodesic curvatures, $1 / \mathfrak{r}$ and $1 / \mathfrak{r}^{\prime}$, of the directed curves $C$ and $\mathcal{C}^{\prime}$. They are

$$
\begin{aligned}
& \frac{1}{\mathfrak{r}}=-\epsilon^{\prime} r r^{\prime} \sin \omega\left[\frac{\nabla}{\nabla s} \frac{\cot \omega}{r^{\prime}}+\frac{\nabla}{\nabla s^{\prime}} \frac{\csc \omega}{r}\right], \\
& \frac{1}{\mathfrak{r}^{\prime}}=\epsilon r r^{\prime} \sin \omega\left[\frac{\nabla}{\nabla s} \frac{\csc \omega}{r^{\prime}}+\frac{\nabla}{\nabla s^{\prime}} \frac{\cot \omega}{r}\right] .
\end{aligned}
$$

But it may be shown that the Codazzi equations, expressed in invariant form with reference to the curves $C$ and $C^{\prime}, \dagger$ may be written

$$
\frac{\nabla}{\nabla s} \frac{\cot \omega}{r^{\prime}}+\frac{\nabla}{\nabla s^{\prime}} \frac{\csc \omega}{r}=-\frac{1}{r^{\prime}} \frac{1}{a}, \quad \frac{\nabla}{\nabla s} \frac{\csc \omega}{r^{\prime}}+\frac{\nabla}{\nabla s^{\prime}} \frac{\cot \omega}{r}=\frac{1}{r} \frac{1}{a^{\prime}},
$$

where $1 / a$ and $1 / a^{\prime}$ denote, as usual, the angular spreads of the families of curves $C^{\prime}$ and $C$ with respect to one another.

Hence

$$
\frac{1}{r} \frac{1}{\mathfrak{r}}=\epsilon^{\prime} \frac{\sin \omega}{a}, \quad \frac{1}{r^{\prime}} \frac{1}{\mathfrak{r}^{\prime}}=\epsilon \frac{\sin \omega}{a^{\prime}} .
$$

It is evident, from the deduction of these equations, that they are equivalent to the equations of Codazzi. Hence, we have obtained simple geometric interpretations of what are ordinarily rather complicated equations.

In order to compute the angular spreads, with respect to one another, of the families of curves $\mathcal{C}$ and $\mathcal{C}^{\prime}$ on the sphere, we must first agree on a positive direction of rotation for the measurement of angles on the sphere. It is customary to take, as the positive direction of rotation about a point $P$ on the sphere, the direction which is counterclockwise when the sphere is viewed from the exterior, e.g., from the tip of the vector $\zeta$ normal to it at $P$. It turns out, then, that to the positive direction of rotation about a point $P$ on $S$ corresponds the positive or negative direction of rotation about the corresponding point $P$ on the sphere according as $K>0$ or $K<0$.

For present purposes, it is more convenient to choose the positive direction of rotation about the point $P$ on the sphere so that the numerically smallest directed angle, $\Omega$, from the directed curve $C$ through $P$ to the directed curve $\mathcal{C}^{\prime}$ through $P$ is positive, that is, so that the positive direction

$\dagger$ Belgian memoir, loc. cit., p. 84, equations (96). 
of rotation about $P$ always corresponds to the positive direction of rotation about $P$. Evidently, this direction about $P$, in order to appear counterclockwise, must be viewed from the tip of the vector $z=\epsilon \epsilon^{\prime} \zeta$ at $P$, that is, from the exterior of the sphere if $\epsilon \epsilon^{\prime}=1$, and from the interior if $\epsilon \epsilon^{\prime}=-1$.

Since $\Omega(0<\Omega<\pi)$ is the angle at $\mathcal{P}$ from $\gamma$ to $\gamma^{\prime}$,

$$
\cos \Omega=-\epsilon \epsilon^{\prime} \cos \omega, \sin \Omega=\sin \omega \text {. }
$$

Consequently, $\Omega=\pi-\omega$ if $\epsilon \epsilon^{\prime}=1$ and $\Omega=\omega$ if $\epsilon \epsilon^{\prime}=-1$. Thus, in any case,

$$
d \Omega=-\epsilon \epsilon^{\prime} d \omega \text {. }
$$

From (48) and (49), we find, for the elements of arc, $d \sigma$ and $d \sigma^{\prime}$, of the directed curves $\mathcal{C}$ and $\mathcal{C}^{\prime}$, the values

$$
d \sigma=\frac{\epsilon}{r} \csc \omega d s, \quad d \sigma^{\prime}=\frac{\epsilon^{\prime}}{r^{\prime}} \csc \omega d s^{\prime} .
$$

Hence,

$$
\frac{\partial \Omega}{\partial \sigma}=-\epsilon^{\prime} r \sin \omega \frac{\partial \omega}{\partial s}, \quad \frac{\partial \Omega}{\partial \sigma^{\prime}}=-\epsilon r^{\prime} \sin \omega \frac{\partial \omega}{\partial s^{\prime}},
$$

where $\partial / \partial \sigma$ and $\partial / \partial \sigma^{\prime}$ denote directional differentiation in the positive directions of the curves $C$ and $\mathcal{C}^{\prime}$, respectively.

By means of these formulas and equations (50), we obtain the angular spread, $1 / A$, of the curves $\mathcal{C}^{\prime}$ with respect to the curves $\mathcal{C}$ and the angular spread, $1 / A^{\prime}$, of the curves $C$ with respect to the curves $\bigodot^{\prime}$ :

$$
\frac{\epsilon^{\prime}}{r} \frac{1}{A}=\frac{\sin \omega}{\rho}, \quad \frac{\epsilon}{r^{\prime}} \frac{1}{A^{\prime}}=\frac{\sin \omega}{\rho^{\prime}} .
$$

It is to be noted that equations (50) and (51) exhibit a reciprocity between the conjugate system on the surface and the system representing it on the sphere. The two sets of equations imply the following theorems.

THEOREM 22. The curves of one of two conjugate families of curves on a surface are parallel with respect to the curves of the second family if and only if the curves of the second family are represented on the sphere by geodesics.

THEOREM 23. The curves of one family of a system of curves on the sphere which represents a conjugate system of curves on the surface are parallel with respect to the curves of the second family if and only if the curves on the surface represented by the curves of the second family are geodesics.

From equations (51) we also conclude the theorem of Voss to the effect that a conjugate system on a surface consists of geodesics when and only when it is represented by a system of curves on the Gauss sphere which clothes the 
sphere. The reciprocal theorem, guaranteed by equations (50), says that a surface is a translation surface if and only if there exists on it a conjugate system whose curves are represented on the sphere by geodesics.

Let $1 / B$ be the distantial spread of the curves $C^{\prime}$ with respect to the curves $C$, and $1 / B^{\prime}$, that of the curves $C$ with respect to the curves $\mathcal{C}^{\prime}$. According to equations (14), we have

$$
\frac{\sin \Omega}{B}=\frac{1}{A}-\frac{\cos \Omega}{A^{\prime}}, \quad \frac{\sin \Omega}{B^{\prime}}=\frac{1}{A^{\prime}}-\frac{\cos \Omega}{A} .
$$

Substituting for $\Omega$ its value in terms of $\omega$ and for $1 / A, 1 / A^{\prime}$ their values from (51), we obtain

$$
\frac{\epsilon^{\prime}}{B}=\frac{r}{\rho}+\frac{r^{\prime}}{\rho^{\prime}} \cos \omega, \quad \frac{\epsilon}{B^{\prime}}=\frac{r}{\rho} \cos \omega+\frac{r^{\prime}}{\rho^{\prime}} .
$$

The corresponding formulas for the distantial spreads, $1 / b$ and $1 / b^{\prime}$, of the curves $C^{\prime}$ and $C$ with respect to the curves $C$ and $C^{\prime}$ may be obtained in a similar fashion. They are

$$
\frac{\sin ^{2} \omega}{b}=\frac{\epsilon^{\prime}}{r} \frac{1}{\mathfrak{r}}-\frac{\epsilon}{r^{\prime}} \frac{\cos \omega}{\mathfrak{r}^{\prime}}, \quad \frac{\sin ^{2} \omega}{b^{\prime}}=-\frac{\epsilon^{\prime}}{r} \frac{\cos \omega}{\mathfrak{r}}+\frac{\epsilon}{r^{\prime}} \frac{1}{\mathfrak{r}^{\prime}} .
$$

For a conjugate system which does not consist of the lines of curvature on the surface, we readily deduce the following results.

THEOREM 24. If two of the four quantities $1 / \rho, 1 / \rho^{\prime}, 1 / B, 1 / B^{\prime}$ are zero, then all four are zero and the conjugate system consists of geodesics.

TheOREM 25. If two of the four quantities $1 / \mathfrak{r}, 1 / \mathfrak{r}^{\prime}, 1 / b, 1 / b^{\prime}$ are zero, then all four are zero and the conjugate system clothes the surface.

Lines of curvature. If $\omega=\pi / 2$, then $\Omega=\pi / 2$ and $1 / a=1 / b=1 / \rho, 1 / a^{\prime}$ $=1 / b^{\prime}=1 / \rho^{\prime}$, and $1 / A=1 / B=1 / \mathfrak{r}, 1 / A^{\prime}=1 / B^{\prime}=1 / \mathfrak{r}^{\prime}$. In this case, equations $(50)$ to $(53)$ all reduce to the single known pair of equations $\dagger$

$$
\frac{\epsilon^{\prime}}{r} \frac{1}{\mathfrak{r}}=\frac{1}{\rho}, \quad \frac{\epsilon}{r^{\prime}} \frac{1}{\mathfrak{r}^{\prime}}=\frac{1}{\rho^{\prime}} .
$$

15. Spherical representation of the asymptotic lines. We assume now that the surface $S$ is a surface of negative curvature and that the curves $C$ and $C^{\prime}$ are its asymptotic lines. Then $K=-1 / \tau^{2}$, where $1 / \tau$ and $-1 / \tau$ are respectively the geodesic torsions of the directed curves $C$ and $C^{\prime}$, and formula (46) for the geodesic curvature of a family of curves on the sphere becomes

$\dagger$ Belgian memoir, loc. cit., p. 37. 


$$
\frac{\sin \omega}{\mathfrak{r}}=\tau^{2}\left[\frac{\nabla}{\nabla s}\left(\frac{\partial \zeta}{\partial s^{\prime}} \mid \gamma\right)-\frac{\nabla}{\nabla s^{\prime}}\left(\frac{\partial \zeta}{\partial s} \mid \gamma\right)\right]
$$

In this case $\dagger$

$$
\frac{\partial \zeta}{\partial s}=\frac{1}{\tau} \bar{\xi}, \quad \frac{\partial \zeta}{\partial s^{\prime}}=-\frac{1}{\tau} \bar{\xi}^{\prime}
$$

where the vectors $\bar{\xi}, \bar{\xi}^{\prime}$ are as defined in $\$ 14$. Hence, the unit vectors tangent to the directed curves $\mathcal{C}$ and $\mathcal{C}^{\prime}$ on the sphere which represent respectively the curves $C$ and $C^{\prime}$ are

$$
\gamma=\epsilon \bar{\xi}, \gamma^{\prime}=-\epsilon \bar{\xi}^{\prime}
$$

where $\epsilon=1$ if $1 / \tau>0$ and $\epsilon=-1$ if $1 / \tau<0$.

By means of the foregoing formulas, we find for the geodesic curvatures of the curves $C$ and $\mathcal{C}^{\prime}$ the values

$$
\frac{\sin \omega}{\mathfrak{r}}=-\epsilon \tau^{2}\left[\frac{\nabla}{\nabla s} \frac{\cos \omega}{\tau}+\frac{\nabla}{\nabla s^{\prime}} \frac{1}{\tau}\right], \frac{\sin \omega}{\mathfrak{r}^{\prime}}=\epsilon \tau^{2}\left[\frac{\nabla}{\nabla s} \frac{1}{\tau}+\frac{\nabla}{\nabla s^{\prime}} \frac{\cos \omega}{\tau}\right] .
$$

But the Codazzi equations, expressed in forms referred to the asymptotic lines $C$ and $C^{\prime}, \ddagger$ may be written

$$
\frac{\nabla}{\nabla s} \frac{\cos \omega}{\tau}+\frac{\nabla}{\nabla s^{\prime}} \frac{1}{\tau}=\frac{\sin \omega}{\rho} \frac{1}{\tau}, \quad \frac{\nabla}{\nabla s} \frac{1}{\tau}+\frac{\nabla}{\nabla s^{\prime}} \frac{\cos \omega}{\tau}=-\frac{\sin \omega}{\rho^{\prime}} \frac{1}{\tau} .
$$

Hence we obtain, in the present case, the following geometric forms of the Codazzi equations:

$$
\frac{\epsilon}{\tau} \frac{1}{\mathfrak{r}}=-\frac{1}{\rho}, \frac{\epsilon}{\tau} \frac{1}{\mathfrak{r}^{\prime}}=-\frac{1}{\rho^{\prime}} .
$$

If $z, \Omega, d \sigma$, and $d \sigma^{\prime}$ are defined as in $\$ 14$, we find that $z=-\zeta, \Omega=\pi-\omega$, and

$$
d \sigma=\frac{\epsilon}{\tau} d s, \quad d \sigma^{\prime}=\frac{\epsilon}{\tau} d s^{\prime}
$$

Hence

$$
\frac{\epsilon}{\tau} \frac{\partial \Omega}{\partial \sigma}=-\frac{\partial \omega}{\partial s}, \quad \frac{\epsilon}{\tau} \frac{\partial \Omega}{\partial \sigma^{\prime}}=-\frac{\partial \omega}{\partial s^{\prime}} .
$$

† Belgian memoir, loc. cit., p. 77.

$\ddagger$ Belgian memoir, loc. cit., p. 86 . 
From these equations and (54), we obtain for the angular spreads of the curves $\mathcal{C}^{\prime}$ and $\mathcal{C}$ with respect to the curves $\mathcal{C}$ and $\mathcal{C}^{\prime}$ the values

$$
\frac{\epsilon}{\tau} \frac{1}{A}=-\frac{1}{a}, \quad \frac{\epsilon}{\tau} \frac{1}{A^{\prime}}=-\frac{1}{a^{\prime}} .
$$

Equations (54) tell us that an asymptotic line is a geodesic and hence a straight line if and only if the curve representing it on the sphere is a geodesic on the sphere,-a fact which is obvious geometrically. But the equations go further. They say that, if the asymptotic line is not a straight line, the ratio of its geodesic curvature to the geodesic curvature of the curve representing it on the sphere is the negative of the numerical value of its geodesic torsion. In particular, then, the two geodesic curvatures are opposite in sign.

From equation (55) follows

THEOREM 26. One family of asymptotic lines on a surface is parallel with respect to the second family if and only if the family of curves on the sphere which represents the first family is parallel with respect to the family of curves which represents the second.

A corollary to this theorem consists in the known fact that the asymptotic lines clothe the surface when and only when the curves which represent them on the sphere clothe the sphere.

When we substitute for $1 / a, 1 / a^{\prime}, 1 / A, 1 / A^{\prime}$ in equations (55) their values in terms of $1 / b, 1 / b^{\prime}, 1 / B, 1 / B^{\prime}$, we obtain the equations

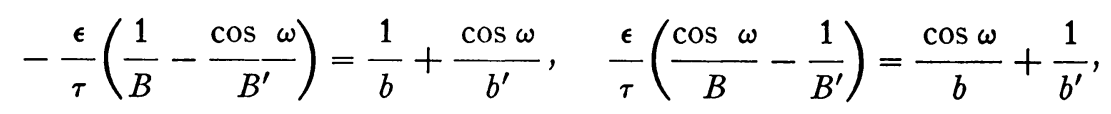

which may be solved for $1 / B, 1 / B^{\prime}$ in terms of $1 / b, 1 / b^{\prime}$, or vice versa.

From equations (54) and (55) we conclude

$$
\frac{1}{\rho}: \frac{1}{\mathfrak{r}}=\frac{1}{\rho^{\prime}}: \frac{1}{\mathfrak{r}^{\prime}}=\frac{1}{a}: \frac{1}{A}=\frac{1}{a^{\prime}}: \frac{1}{A^{\prime}} .
$$

Corresponding to these relations we have, by virtue of (56), the following facts: if $1 / b=1 / b^{\prime}$, then $1 / B=1 / B^{\prime}$, and conversely; and, if $1 / b=-1 / b^{\prime}$, then $1 / B=-1 / B^{\prime}$, and conversely.

THEOREM 27. If two of the four quantities $1 / b, 1 / b^{\prime}, 1 / B, 1 / B^{\prime}$ for the asymptotic lines of a nonminimal surface of negative curvature are zero, then all four are zero and the asymptotic lines clothe the surface.

In the case of a minimal surface, $\omega=\pi / 2$ and $\Omega=\pi / 2$, and equations (55) and (56) both reduce to equations (54). 
16. Spherical representation of an arbitrary system of curves. We shall find it useful here to introduce the characteristic vectors associated with the given curves $C$ and $C^{\prime}$. The characteristic vectors associated with a given directed curve are unit vectors in the points of the curve which have the directions conjugate to those of the curve and are so oriented that the smallest positive directed angle at a point $P$ of the curve from the positive direction of the curve at $P$ to the characteristic vector at $P$ lies in the interval $0 \leqq \theta<\pi$.

Let $\alpha$ and $\alpha^{\prime}$ be the characteristic vectors at $P$ associated with the curves $C$ and $C^{\prime}$ which pass through $P$, and let $\psi$ and $\psi^{\prime}$ be respectively the directed angles from $\xi$ and $\xi^{\prime}$ (the unit vectors tangent at $P$ to $C$ and $C^{\prime}$ ) to $\alpha$ and $\alpha^{\prime}$. Then

$$
\alpha=\xi \cos \psi+\bar{\xi} \sin \psi, \alpha^{\prime}=\xi^{\prime} \cos \psi^{\prime}+\bar{\xi}^{\prime} \sin \psi^{\prime},
$$

where $\bar{\xi}$ and $\bar{\xi}^{\prime}$ are the unit vectors in the tangent plane at $P$ advanced by $+\pi / 2$ over $\xi$ and $\xi^{\prime}$, respectively.

Since the directions of $\alpha$ and $\alpha^{\prime}$ are conjugate to those of $C$ and $C^{\prime}$, they coincide respectively with the directions of the vectors $\partial \zeta / \partial \widetilde{s \zeta}$ and $\partial \zeta / \partial \widetilde{s}^{\prime} \zeta$. But $\dagger$

$$
\frac{\partial \zeta}{\partial s}=-\frac{1}{r} \xi+\frac{1}{\tau} \bar{\xi}, \quad \frac{\partial \zeta}{\partial s^{\prime}}=-\frac{1}{r^{\prime}} \xi^{\prime}+\frac{1}{\tau^{\prime}} \overline{\xi^{\prime}} .
$$

The directions of $\alpha$ and $\alpha^{\prime}$ are, then, the same as those of the vectors

$$
\frac{1}{\tau} \xi+\frac{1}{r} \bar{\xi}, \quad \frac{1}{\tau^{\prime}} \xi^{\prime}+\frac{1}{r^{\prime}} \overline{\xi^{\prime}} .
$$

Consequently, when we introduce the "total normal curvatures"

$$
\frac{1}{\kappa}=\left(\frac{1}{r^{2}}+\frac{1}{\tau^{2}}\right)^{1 / 2}, \frac{1}{\kappa^{\prime}}=\left(\frac{1}{r^{\prime 2}}+\frac{1}{\tau^{\prime 2}}\right)^{1 / 2}
$$

of the curves $C$ and $C^{\prime}$, we have

$$
\begin{array}{ll}
\frac{1}{r}=\frac{\epsilon}{\kappa} \sin \psi, & \frac{1}{r^{\prime}}=\frac{\epsilon^{\prime}}{\kappa^{\prime}} \sin \psi^{\prime}, \\
\frac{1}{\tau}=\frac{\epsilon}{\kappa} \cos \psi, & \frac{1}{\tau^{\prime}}=\frac{\epsilon^{\prime}}{\kappa^{\prime}} \cos \psi^{\prime},
\end{array}
$$

where, if $\psi \neq 0, \epsilon= \pm 1$ according as $1 / r_{<}^{>} 0$, and, if $\psi=0, \epsilon= \pm 1$ according as $1 / \tau_{<} \geq$, and $\epsilon^{\prime}$ is similarly defined.

$\dagger$ Belgian memoir, loc. cit., p. 77. 
The expressions (58) for $\partial \zeta / \partial s$ and $\partial \zeta / \partial s^{\prime}$ now take the forms

(61) $\frac{\partial \zeta}{\partial s}=-\frac{\epsilon}{\kappa}(\xi \sin \psi-\bar{\xi} \cos \psi), \quad \frac{\partial \zeta}{\partial s^{\prime}}=-\frac{\epsilon^{\prime}}{\kappa^{\prime}}\left(\xi^{\prime} \sin \psi^{\prime}-\bar{\xi}^{\prime} \cos \psi^{\prime}\right)$.

The characteristic vectors $\alpha$ and $\alpha^{\prime}$ are evidently perpendicular, respectively, to the vectors $\partial \zeta / \partial s$ and $\partial \zeta / \partial s^{\prime}$. But

$$
\widetilde{\alpha} \frac{\partial \zeta}{\partial s}=\frac{\epsilon}{\kappa} \zeta, \quad \widetilde{\alpha^{\prime}} \frac{\partial \zeta}{\partial s^{\prime}}=\frac{\epsilon^{\prime}}{\kappa^{\prime}} \zeta^{\prime} .
$$

Hence, the angle from $\alpha$ to $\partial \zeta / \partial s$ is $\epsilon \pi / 2$ and that from $\alpha^{\prime}$ to $\partial \zeta / \partial s^{\prime}$ is $\epsilon^{\prime} \pi / 2$.

It follows that the angle from $\xi$ to $\partial \zeta / \partial s$ is $\psi+\epsilon \pi / 2$ and that the angle from $\xi^{\prime}$ to $\partial \zeta / \partial s^{\prime}$ is $\psi^{\prime}+\epsilon^{\prime} \pi / 2$. Incidentally, these results involve the well known fact that a direction at a point $\mathscr{P}$ of the Gauss sphere corresponding to a given direction at the corresponding point $P$ of the surface is perpendicular to the direction at $P$ which is conjugate to the given direction.

It is now evident that the angle on the surface from $\partial \zeta / \partial s$ to $\partial \zeta / \partial s^{\prime}$ is $-(\psi+\epsilon \pi / 2)+\omega+\left(\psi^{\prime}+\epsilon^{\prime} \pi / 2\right)$. Hence the angle, $\Omega$, on the sphere from $\partial \zeta / \partial s$ to $\partial \zeta / \partial s^{\prime}$, as seen from the tip of the vector $z=\eta \zeta$, is

$$
\Omega=\eta\left(\omega+\psi^{\prime}-\psi+\left(\epsilon^{\prime}-\epsilon\right) \frac{\pi}{2}\right)
$$

where, as usual, $\eta= \pm 1$ according as $K^{>}<0$. Hence

$$
\cos \Omega=\epsilon \epsilon^{\prime} \cos \left(\omega+\psi^{\prime}-\psi\right), \sin \Omega=\eta \epsilon \epsilon^{\prime} \sin \left(\omega+\psi^{\prime}-\psi\right) .
$$

We next recall the identity $\dagger$

$$
\frac{\cos \omega}{r}-\frac{\sin \omega}{\tau}=\frac{\cos \omega}{r^{\prime}}+\frac{\sin \omega}{\tau^{\prime}} .
$$

Denoting the common value of these two expressions by $P$ and computing each in terms of $1 / \kappa, 1 / \kappa^{\prime}, \psi, \psi^{\prime}$, $\omega$, we find

$$
P=\frac{\epsilon}{\kappa} \sin (\psi-\omega), \text { or } P=\frac{\epsilon^{\prime}}{\kappa^{\prime}} \sin \left(\psi^{\prime}+\omega\right) \text {. }
$$

Thus, the five quantities fundamental in our discussion are related by the identity

$$
\frac{\epsilon}{\kappa} \sin (\psi-\omega)=\frac{\epsilon^{\prime}}{\kappa^{\prime}} \sin \left(\psi^{\prime}+\omega\right)
$$

$\dagger$ Belgian memoir, loc. cit., p. 74. 
For the total curvature $K$ of the surface we have the formula $\dagger$

$$
K \sin ^{2} \omega=\frac{1}{r} \frac{1}{r^{\prime}}-P^{2}
$$

which, by means of $(60),(63)$, and (62), can be reduced to the simpler form

$$
K=\frac{\eta}{\kappa \kappa^{\prime}} \frac{\sin \Omega}{\sin \omega} .
$$

Substituting the value of $K$ into equation (46), we obtain, as the formula for the geodesic curvature of the curves on the Gauss sphere with the unit tangent vector $\gamma$,

$$
\frac{\sin \Omega}{\mathfrak{r}}=\kappa \kappa^{\prime}\left[\frac{\nabla}{\nabla s}\left(\frac{\partial \zeta}{\partial s^{\prime}} \mid \gamma\right)-\frac{\nabla}{\nabla s^{\prime}}\left(\frac{\partial \zeta}{\partial s} \mid \gamma\right)\right] .
$$

According to equations (61), the unit vectors tangent respectively to the curves $\mathcal{C}$ and $\mathcal{C}^{\prime}$ on the sphere which represent the given curves $C$ and $C^{\prime}$ are

$$
\gamma=-\epsilon(\xi \sin \psi-\bar{\xi} \cos \psi), \gamma^{\prime}=-\epsilon^{\prime}\left(\xi^{\prime} \sin \psi^{\prime}-\bar{\xi}^{\prime} \cos \psi^{\prime}\right) .
$$

Hence we find, as the geodesic curvatures of the curves $\mathcal{C}$ and $\mathcal{C}^{\prime}$,

$$
\frac{\sin \Omega}{\mathfrak{r}}=\kappa \kappa^{\prime}\left[\frac{\nabla}{\nabla s} \frac{\cos \Omega}{\kappa^{\prime}}-\frac{\nabla}{\nabla s^{\prime}} \frac{1}{\kappa}\right], \quad \frac{\sin \Omega}{\mathfrak{r}^{\prime}}=\kappa \kappa^{\prime}\left[\frac{\nabla}{\nabla s} \frac{1}{\kappa^{\prime}}-\frac{\nabla}{\nabla s^{\prime}} \frac{\cos \Omega}{\kappa}\right] \text {. }
$$

We turn now to the Codazzi equations. $f$ These equations, when expressed in terms of $1 / \kappa, 1 / \kappa^{\prime}, \psi, \psi^{\prime}, \omega$, take the forms

$\epsilon \frac{\nabla}{\nabla s^{\prime}} \frac{\sin \psi}{\kappa}-\epsilon^{\prime} \frac{\nabla}{\nabla s} \frac{\sin \left(\psi^{\prime}+\omega\right)}{\kappa^{\prime}}+\epsilon\left(\frac{1}{\rho^{\prime}}-\frac{\partial \omega}{\partial s^{\prime}}\right) \frac{\cos \psi}{\kappa}-\frac{\epsilon^{\prime}}{\rho} \frac{\cos \left(\psi^{\prime}+\omega\right)}{\kappa^{\prime}}=0$,

$\epsilon \frac{\nabla}{\nabla s^{\prime}} \frac{\sin (\psi-\omega)}{\kappa}-\epsilon^{\prime} \frac{\nabla}{\nabla s} \frac{\sin \psi^{\prime}}{\kappa^{\prime}}+\frac{\epsilon}{\rho^{\prime}} \frac{\cos (\psi-\omega)}{\kappa}-\epsilon^{\prime}\left(\frac{1}{\rho}+\frac{\partial \omega}{\partial s}\right) \frac{\cos \psi^{\prime}}{\kappa^{\prime}}=0$.

When we expand the derivatives of the products such as $(\sin \psi) / \kappa$, applying the modified derivative always to the total normal curvature involved, we get two equations in $\left(\nabla / \nabla s^{\prime}\right)(1 / \kappa),(\nabla / \nabla s)\left(1 / \kappa^{\prime}\right), 1 / \kappa$, and $1 / \kappa^{\prime}$. Eliminating in turn the terms in $1 / \kappa$ and $1 / \kappa^{\prime}$ from these equations, we obtain the equations

$\dagger$ Belgian memoir, loc. cit., p. 75.

$\ddagger$ Belgian memoir, loc. cit., p. 80 , equations (93). 


$$
\begin{aligned}
& \frac{\nabla}{\nabla s} \frac{\cos \Omega}{\kappa^{\prime}}-\frac{\nabla}{\nabla s^{\prime}} \frac{1}{\kappa}=\eta\left(\frac{1}{\rho}+\frac{\partial \psi}{\partial s}\right) \frac{\sin \Omega}{\kappa^{\prime}}, \\
& \frac{\nabla}{\nabla s} \frac{1}{\kappa^{\prime}}-\frac{\nabla}{\nabla s^{\prime}} \frac{\cos \Omega}{\kappa}=\eta\left(\frac{1}{\rho^{\prime}}+\frac{\partial \psi^{\prime}}{\partial s^{\prime}}\right) \frac{\sin \Omega}{\kappa},
\end{aligned}
$$

which, by virtue of the expressions for $1 / \mathfrak{r}$ and $1 / \mathfrak{r}^{\prime}$, take the simple forms

$$
\frac{\eta}{\kappa} \frac{1}{\mathfrak{r}}=\frac{1}{\rho}+\frac{\partial \psi}{\partial s}, \quad \frac{\eta}{\kappa^{\prime}} \frac{1}{\mathfrak{r}^{\prime}}=\frac{1}{\rho^{\prime}}+\frac{\partial \psi^{\prime}}{\partial s^{\prime}} .
$$

It is to be noted that $1 / \rho+\partial \psi / \partial s$ is the angular spread, with respect to the curves $C$, of the curves which are conjugate to the curves $C$, and that $1 / \rho^{\prime}$ $+\partial \psi^{\prime} / \partial s^{\prime}$ has a similar meaning.

It follows from (61) and (65) that the elements of arc of the directed curves $\mathcal{C}$ and $\mathcal{C}^{\prime}$ are

$$
d \sigma=\frac{d s}{\kappa}, \quad d \sigma^{\prime}=\frac{d s^{\prime}}{\kappa^{\prime}} .
$$

From these relations and equation (62a) we get

$$
\frac{\eta}{\kappa} \frac{\partial \Omega}{\partial \sigma}=\frac{\partial\left(\omega+\psi^{\prime}-\psi\right)}{\partial s}, \quad \frac{\eta}{\kappa^{\prime}} \frac{\partial \Omega}{\partial \sigma^{\prime}}=\frac{\partial\left(\omega+\psi^{\prime}-\psi\right)}{\partial s^{\prime}} .
$$

Hence we obtain, as the values of the angular spreads, with respect to one another, of the curves $\mathcal{C}$ and $\mathcal{C}^{\prime}$,

$$
\frac{\eta}{\kappa} \frac{1}{A}=\frac{1}{\rho}+\frac{\partial\left(\psi^{\prime}+\omega\right)}{\partial s}, \quad \frac{\eta}{\kappa^{\prime}} \frac{1}{A^{\prime}}=\frac{1}{\rho^{\prime}}+\frac{\partial(\psi-\omega)}{\partial s^{\prime}} .
$$

The right-hand side of the first equation is the angular spread, with respect to the curves $C$, of the curves which are conjugate to the curves $C^{\prime}$, and the right-hand side of the second equation has a similar geometric interpretation.

When equations (66) are solved for $1 / \rho$ and $1 / \rho^{\prime}$ in terms of $1 / \mathfrak{r}$ and $1 / \mathfrak{r}^{\prime}$, we get

$$
\frac{1}{\rho}=\frac{\eta}{\kappa}\left(\frac{1}{\mathfrak{r}}-\eta \frac{\partial \psi}{\partial \sigma}\right), \quad \frac{1}{\rho^{\prime}}=\frac{\eta}{\kappa^{\prime}}\left(\frac{1}{\mathfrak{r}^{\prime}}-\eta \frac{\partial \psi^{\prime}}{\partial \sigma^{\prime}}\right) .
$$

The angle on the sphere from $\gamma$ to $\xi$, as viewed from the tip of $z$, is $-\eta(\psi$ $+\epsilon \pi / 2)$, and the directions on the sphere orthogonal to those of the vectors $\xi$ are tangent to the curves on the sphere representing the curves conjugate to the curves $C$. Hence, the expression $1 / \mathfrak{r}-\eta \partial \psi / \partial \sigma$ is the angular spread, with respect to the curves $C$, of the curves on the sphere which represent the curves conjugate to the curves $C$. 
From equations (68) we readily obtain the values of the angular spreads, with respect to one another, of the curves $C$ and $C^{\prime}$, namely,

$$
\frac{1}{a}=\frac{\eta}{\kappa}\left(\frac{1}{\mathfrak{r}}-\eta \frac{\partial(\psi-\omega)}{\partial \sigma}\right), \quad \frac{1}{a^{\prime}}=\frac{\eta}{\kappa^{\prime}}\left(\frac{1}{\mathfrak{r}^{\prime}}-\eta \frac{\partial\left(\psi^{\prime}+\omega\right)}{\partial \sigma^{\prime}}\right) .
$$

The angle on the sphere from $\gamma$ to $\xi^{\prime}$ is $\eta(\omega-(\psi+\epsilon \pi / 2))$. But the directions on the sphere orthogonal to those of the vectors $\xi^{\prime}$ are tangent to the curves on the sphere which represent the curves conjugate to the curves $C^{\prime}$. Hence, the expression in the parenthesis on the right-hand side of the first equation is the angular spread, with respect to the curves $\mathcal{C}$, of the curves on the sphere representing the curves conjugate to the curves $C^{\prime}$.

We shall state the results implied in formulas (66) to (69) in terms of an arbitrary family of curves $C$ on the surface and the corresponding family of curves $C$ on the sphere. From (69) and (67) we have

TheOREM 28. A family of curves on the surface consists of parallel curves with respect to the curves $C$ if and only if the conjugate family of curves is represented on the sphere by curves which are parallel with respect to the curves $\mathcal{C}$. Or, a family of curves on the sphere consists of parallel curves with respect to the curves $\mathcal{C}$ if and only if the family conjugate to the family which it represents on the surface consists of curves which are parallel with respect to the curves $C$.

From (68) and (66), we obtain

THEOREM 29. The curves $C$ are geodesics on the surface if and only if the curves conjugate to them are represented on the sphere by curves which are parallel with respect to the curves $\mathcal{C}$. The curves $C$ are geodesics on the sphere if and only if the curves on the surface which are conjugate to the curves $C$ are parallel with respect to the curves $C$.

From Theorem 28 we conclude

THEOREM 30. Two families of curves clothe the surface if and only if the family of curves conjugate to each of them is represented by curves on the sphere which are parallel with respect to the curves which represent the other family.

There is, of course, a reciprocal theorem concerning the clothure of the sphere.

It is evident that Theorems (28), (29), (30) include, either directly or indirectly, the theorems bearing on the spherical representations of conjugate systems and asymptotic lines. Moreover, it is not difficult to show that our present formulas reduce to those of $\$ 14$ when $\psi=\omega$ and $\psi^{\prime}=\pi-\omega$, and become those of $\$ 15$ when $\psi=0$ and $\psi^{\prime}=0$. 
Inasmuch as Theorems 29 and 30 are obtainable from Theorem 28, the latter theorem is the fundamental theorem including all the others.

We proceed to put this theorem in a second, and perhaps more striking, form. Returning to the first of the equations (69), we note that the left-hand side is the angular spread of the vector field $\xi^{\prime}$ on the surface with respect to the curves $C$, and that the expression in the parenthesis on the right-hand side is the angular spread of the vector field $\xi^{\prime}$ on the sphere with respect to the curves $\mathcal{C}$.

TheOREm 31. Directions at the points of a curve on the surface are parallel with respect to the curve if and only if the same directions at the corresponding points of the corresponding curve on the sphere are parallel with respect to this curve.

We turn finally to the relationships between the distantial spreads of the two families of curves on the surface with respect to one another and the distantial spreads, with respect to each other, of the two corresponding families of curves on the sphere. These are found to be

$$
\begin{aligned}
& \frac{\eta}{\kappa}\left(\frac{1}{B}+\frac{\cos \Omega}{B^{\prime}}\right)=\frac{\sin \Omega}{\sin \omega}\left(\frac{1}{b}+\frac{\cos \omega}{b^{\prime}}+\sin \omega \frac{\partial \psi^{\prime}}{\partial s}\right), \\
& \frac{\eta}{\kappa^{\prime}}\left(\frac{\cos \Omega}{B}+\frac{1}{B^{\prime}}\right)=\frac{\sin \Omega}{\sin \omega}\left(\frac{\cos \omega}{b}+\frac{1}{b^{\prime}}+\sin \omega \frac{\partial \psi}{\partial s^{\prime}}\right) .
\end{aligned}
$$

It is evident from these relations that, if $1 / b=0$ and $1 / b^{\prime}=0$, then $1 / B=0$ and $1 / B^{\prime}=0$ if and only if $\partial \psi^{\prime} / \partial s=0$ and $\partial \psi / \partial s^{\prime}=0$.

THEOREM 32.Two families of curves which clothe the surface are represented on the sphere by two families of curves which clothe the sphere if and only if the angle under which each family of curves is cut by the family of curves conjugate to it is constant along each curve of the other family.

The same conditions are necessary and sufficient that two families of curves clothe the surface when it is known that the families representing them on the sphere clothe the sphere.

HARVARD UNIVERSITY.

Cambridge, Mass. 